\title{
Current advances in molecular, biochemical, and computational modeling analysis of microalgal triacylglycerol biosynthesis
}

3 5

6

Sangram K. Lenka ${ }^{\mathrm{a}, 1}$, Nicole Carbonaro ${ }^{\mathrm{b}, 1}$, Rudolph Park ${ }^{\mathrm{c}}$, Stephen M. Miller ${ }^{\mathrm{c}, *}$, Ian Thorpe ${ }^{\mathrm{b}, *}$, and Yantao $\mathrm{Li}^{\mathrm{a}}{ }^{, *}$

${ }^{a}$ Institute of Marine and Environmental Technology, University of Maryland Center for Environmental Science and University of Maryland Baltimore County, Baltimore, Maryland, 21202, USA

${ }^{\mathrm{b}}$ Department of Chemistry and Biochemistry, University of Maryland, Baltimore County, Baltimore, Maryland, 21250, USA

${ }^{\mathrm{c}}$ Department of Biological Sciences, University of Maryland, Baltimore County, Baltimore, Maryland, 21250, USA

.

5

6

(1)

*Corresponding authors: YL: Phone, +1 410-234-8833; E-mail: yantao@umces.edu

8

$$
\begin{aligned}
& \text { IT: Phone: +1 410-455-5728; Email: } \underline{\text { ithorpe@umbc.edu }} \\
& \text { SM: Phone: +1 410-455-3482; Email: stmiller@umbc.edu }
\end{aligned}
$$

${ }^{1}$ These authors contributed equally to this work.

2

3

4

6

7




\section{Abstract}

Triacylglycerols (TAGs) are highly reduced energy storage molecules ideal for biodiesel production. Microalgal TAG biosynthesis has been studied extensively in recent years, both at the molecular level and systems level through experimental studies and computational modeling. However, discussions of the strategies and products of the experimental and modeling approaches are rarely integrated and summarized together in a way that promotes collaboration among modelers and biologists in this field. In this review, we outline advances toward understanding the cellular and molecular factors regulating TAG biosynthesis in unicellular microalgae with an emphasis on recent studies on rate-limiting steps in fatty acid and TAG synthesis, while also highlighting new insights obtained from the integration of multi-omics datasets with mathematical models. Computational methodologies such as kinetic modeling, metabolic flux analysis, and new variants of flux balance analysis are explained in detail. We discuss how these methods have been used to simulate algae growth and lipid metabolism in response to changing culture conditions and how they have been used in conjunction with experimental validations. Since emerging evidence indicates that TAG synthesis in microalgae operates through coordinated crosstalk between multiple pathways in diverse subcellular destinations including the endoplasmic reticulum and plastids, we discuss new experimental studies and models that incorporate these findings for discovering key regulatory checkpoints. Finally, we describe tools for genetic manipulation of microalgae and their potential for future rational algal strain design. This comprehensive review explores the potential synergistic impact of pathway analysis, computational approaches, and molecular genetic manipulation strategies on improving TAG production in microalgae.

Keywords: Microalgae, Lipid, Triacylglycerol biosynthesis, Metabolic Engineering, Computational Modeling, Flux balance analysis, Omics, Biofuels

\section{Introduction}

Climate change, human population growth, and depletion of natural resources impose a serious burden on the planet. Addressing these challenges while concurrently expanding the global economy will require new technologies that generate renewable resources for food, health products, chemicals, and fuels (Georgianna and Mayfield, 2012, Harvey and Pilgrim, 2011). Oleaginous (oil-producing) microalgae hold promise as a sustainable feedstock for each of these needs, as they can accumulate large amounts of triacylglycerol (TAG) under proper conditions (Benemann, 2013, Hu et al., 2008). TAG is a major storage lipid that plays an important role in energy and carbon storage in many microalgae (Chisti, 2007). It is known that TAG synthesis and storage occur in distinct subcellular compartments within microalgae, and recent evidence indicates that TAG is not only a storage molecule but also plays a crucial role in maintaining intracellular lipid homeostasis, proper membrane structure, and cellular function in response to environmental and developmental cues (Holthuis and Menon, 2014, Kohlwein, 2010). Understanding how these functions and processes are coordinated and regulated will arguably be critical for any sort of rational re-design of TAG biosynthesis in microalgae.

Engineering algae to accumulate high levels of TAG while growing rapidly is an ongoing challenge and only limited success has been reached (Li et al., 2010a, Li et al., 2010b, Tsai et al., 
2014). Such efforts benefit from knowledge of the biochemical and molecular mechanisms regulating fatty acid (FA) and TAG biosynthesis, accumulation, and turnover in other organisms, much of it inferred from studies of higher plants. Interestingly, TAG biosynthesis is relatively complicated in species such as Arabidopsis, for which acyl-lipid metabolism and its regulation involve over 600 genes and at least 120 enzymatic reactions in different organs of the plant (LiBeisson et al., 2013). In comparison, in microalgae, TAG biosynthesis and storage occur within a single cell and involve fewer proteins. For instance, based on the JGI v5.5 of the Chlamydomonas reinhardtii genome, there are just 113 genes predicted to be involved in major steps of acyl-lipid metabolism (Li-Beisson et al., 2015) in that organism, and the number of genes involved in FA metabolism plus TCA cycle is inferred to be just 106 in the diatom Phaeodactylum tricornutum (Mühlroth et al., 2013). Clearly there are other interesting and important metabolic and cell biological differences between plants, green algae, and chromophyte algae, including differences in carbohydrate synthesis and glycolytic pathways, and in the arrangements of their thylakoid membranes (Wilhelm et al., 2006). As our focus is on TAG metabolism, these topics are not discussed in detail in this review

Indeed, whole genome sequencing, de novo transcriptomics, and proteomics analysis of algae have accelerated the basic understanding of FA and TAG biosynthesis for strain improvement (Guarnieri et al., 2011, Merchant et al., 2007, Nguyen et al., 2011, Radakovits et al., 2012). With analytical advancements in lipidomics, it is now possible to quantitatively identify hundreds of natural TAGs and their regioisomers from algae (Rezanka et al., 2014). Using such omics and biochemical information from the model alga $C$. reinhardtii, attempts have been made to derive a draft metabolic network using computational modeling and bioinformatics methods (Boyle and Morgan, 2009, May et al., 2009, May et al., 2008). Mathematical and kinetic models have also been developed to study growth parameters and lipid production in green algae (Packer et al., 2010, Tevatia et al., 2012, Yang et al., 2010). Taken together, current developments in molecular genetics and biochemistry, omics (including genomics, transcriptomics, proteomics, metabolomics, and fluxomics, integrative modeling, and genome engineering tools have the potential to enable precise predictive capabilities and rational engineering of algae for high lipid content, see Figure 1 (Jiang et al., 2014, Recht et al., 2014, Reijnders et al., 2014). The above technological advances offer a unique opportunity to understand algal lipid metabolism in a holistic manner.

This review begins with an overview of the current knowledge of TAG production in microalgae, based on biochemical, physiological, multi-omics, and pathway modification studies, with a focus on the role of major enzymes involved in TAG metabolism. Then we discuss computational methods and their use in data analysis and pathway prediction, with an emphasis on the application of these methodologies to algal TAG production. Finally, we offer a perspective on recently developed genetic engineering tools that may be used to rationally engineer algal strains for favorable biofuel traits (Figure 1) and summarize the genetic modifications outcomes, highlighting some strategies that modelers and experimenters could collaboratively investigate.

\section{(Place for Figure 1)}

\section{Fatty acid and triacylglycerol biosynthesis in microalgae}


FAs are amphipathic molecules consisting of a carboxylic acid group at one end and a hydrocarbon group at the other, with length and degree of saturation of the hydrocarbon moiety variable among different FA species. Acyl-lipids derived from FAs, primarily glycerolipids, form the basic structural components of cell membranes (Li-Beisson et al., 2013). The physiologically inert storage lipid TAG has three fatty acyl groups esterified with the glycerol backbone. Many important mechanistic insights into FA biosynthesis in algae were inferred using the basic framework of Arabidopsis lipid metabolism. However, microalgal lipid metabolism differs from plants in at least two important ways (Liu and Benning, 2012). First, in microalgae (but not plants) the plastidic prokaryotic pathway of TAG synthesis and storage plays a more direct role, and second, algae synthesize glycerolipid with distinct acyl groups not present in plants.

Acetyl-CoA is the precursor of de novo lipid synthesis in the chloroplast. Under photoautotrophic conditions photosynthetic efficiency (PE) is critical in controlling the flux of this reaction as the carbon precursor (acetyl-CoA), energy (ATP), and reducing power (NADH and NADPH) are provided by photosynthesis (Figure 2). FA biosynthesis starts with carboxylation of acetyl-CoA to form malonyl-CoA catalyzed by the multifunctional acetyl-CoA carboxylase (ACCase, EC 6.4.1.2) enzyme complex (see Figure 2) (Cronan and Waldrop, 2002). ACCase has been reported to be a control point of FA biosynthesis in plants and algae (Ohlrogge and Jaworski, 1997) but as described later in this review, more recent evidence suggests that ACCase does not catalyze a rate-limiting step in algae. ACCase is under redox regulatory control (Kozaki et al., 2001). The isoforms and functions of algal ACCase were recently reviewed by Huerliman and Heimann (Huerlimann and Heimann, 2013).

In the algal plastid, malonyl-CoA is transferred to acyl carrier protein (ACP) and subsequently undergoes a series of acyl chain elongation reactions to form $\mathrm{C}_{16}$ or $\mathrm{C}_{18}$ products, catalyzed by the multi-subunit enzyme fatty acid synthase (FAS, EC 2.3.1.85). Elongation of the FA chain terminates due to the action of two enzymes. In photosynthetic organisms, one of these is a chloroplast acyltransferase that removes the acyl group from ACP, whereupon the nascent FA is directly transferred from ACP to glycerol-3-phosphate (G-3-P). The other enzyme, acyl-ACP thioesterase, hydrolyzes acyl-ACP and releases the free FAs. Free FAs are then transferred to the outer envelope of the chloroplast and are reactivated to acyl-CoA to form glycerolipids (Li et al., 2013, Ohlrogge and Browse, 1995). FA elongation and desaturation have been covered recently (Bellou et al., 2014, Li et al., 2013) and thus are not discussed in detail in the following section. Instead, we will focus on recent advances in understanding the Kennedy pathway and alternative pathways to TAG synthesis in algae.

\section{(Place for Figure 2)}

\subsection{Regulatory elements of the Kennedy pathway}

The Kennedy pathway is the primary pathway for TAG synthesis in microalgae and plants, where TAG is produced through sequential transfer of acyl groups from acyl-CoA to the different positions of the glycerol-3-phosphate backbone by acyltransferases (Li-Beisson et al., 2013) . Thus, understanding the genetic regulation of the Kennedy pathway is critical for improving TAG production in microalgae.

\subsubsection{Acyltransferases}


Acyltransferases play an important role in defining the final content and acyl composition of TAG. The acyltransferases of the Kennedy pathway have been identified in sequenced algal genomes, including acyl-CoA:glycerol-3-phosphate acyltransferase (GPAT), acylCoA:lysophosphatidic acid acyltransferase (LPAAT), and the acyl-CoA:diacylglycerol acyltransferase (DGAT). Owing to the substrate specificity of ER- or plastidic-localized LPAAT, glycerolipids with a C18 fatty acid in the $s n-2$ position are thought to be "eukaryotic" lipids derived from the ER in plants and algae, whereas glycerolipids with a C16 in the $s n$-2 position are "prokaryotic" lipids assembled in the plastid (Ohlrogge and Browse, 1995).

The first committed step of the Kennedy pathway is catalyzed by GPAT (EC 2.3.1.15), which transfers the FA from the acyl-CoA pool (acyl-ACP in plastids) to the $s n-1$ position of glycerol3-phosphate and produces lysophosphatidic acid (LPA). In contrast to plants, which possess both ER and plastidic GPAT, red algae (Cyanidioschyzon merolae), diatoms (Phaeodactylum tricornutum and Thalassiosira pseudonana), and green algae (Chlamydomonas reinhardtii and Ostreococcus tauri) are predicted to contain only plastid versions of GPAT homologs (Lykidis and Ivanova, 2008). Nannochloropsis oceanica and N. gaditana possess nine putative GPAT and LPAAT isoforms of which only plastidic GPATs are unambiguously annotated (Vieler et al., 2012). Interestingly, overexpression of the GPAT gene from the oleaginous green microalga Lobosphaera incisa in Chlamydomonas results in up to $50 \%$ increase in TAG without compromising growth (Iskandarov et al., 2015). LPAAT transfers the second acyl group into the $s n-2$ position of LPA and converts it into phosphatidic acid (PA). Phosphatidic acid phosphatase (PAP) dephosphorylates PA to $s n$-1,2-diacylglycerol (sn-1,2- DAG). PA and $s n-1,2-D A G$ are subsequently used to synthesize membrane lipids and TAG. Knocking down LPAAT using artificial microRNAs resulted in $\sim 20 \%$ reduction of neutral lipid production in Chlamydomonas (Lv et al., 2013). No genes for extra-plastidic LPAATs have been identified in the genomes of Chlamydomonas or other algae, except one from the Micromonas pusilla spp (Merchant et al., 2012). It is proposed that a divergent protein or broad substrate specificity GPAT performs the function of extra-plastidic LPAAT in algae (Merchant et al., 2012), but experimental validation has yet to be provided.

DGAT catalyzes the last and committed step of TAG assembly by adding an acyl group to the $s n-3$ position of DAG. This is suggested to be a rate-limiting step of TAG biosynthesis in plants and algae (Lung and Weselake, 2006, Zou et al., 1999). Several different types of DGATs have been identified in higher plants. Type-1 DGAT (DGAT1) and Type-2 DGAT (DGAT2) are structurally distinct types of membrane bound acyltransferases intensively studied in plants (Lung and Weselake, 2006, Ohlrogge and Jaworski, 1997). A type-3 soluble DGAT has been identified in the cytosol of peanut (Saha et al., 2006) whereas a bifunctional DGAT/wax ester synthase has been characterized in Acinetobacter calcoaceticus (Kalscheuer and Steinbuchel, 2003). In multiple sequenced algal genomes to date, DGAT genes including DGAT1 and DGAT2 homologs are predicted to be present (Guiheneuf et al., 2011, Merchant et al., 2007, Radakovits et al., 2012, Vieler et al., 2012, Wagner et al., 2010). However, the function and regulation of different $D G A T$ genes toward TAG synthesis is poorly understood. For example, five type-2 $D G A T$ s (named as DGTT in this organism) genes are present in the $C$. reinhardtii genome. In comparison, only one type-2 DGAT is found in Arabidopsis thaliana. It will be interesting to investigate the function of each Chlamydomonas DGTT in TAG biosynthesis within different subcellular compartments. 
Previously, the $C$. reinhardtii DGTT genes were partially characterized through functional complementation in yeast (Bolling and Fiehn, 2005, Boyle et al., 2012, Sanjaya et al., 2013) and heterologous expression in Arabidopsis (Sanjaya et al., 2013), confirming the acyltransferase activity of CrDGTT1, CrDGTT2, or CrDGTT3. However, their substrate specificity towards different fatty acids, particularly the polyunsaturated fatty acids present in high abundance in Chlamydomonas, has not been studied, and no information is available on their activity towards prokaryotic and eukaryotic substrates, i.e., diacylglycerols (DAGs). Recently, we have developed a radiolabel-free in vitro assay to investigate the function of CrDGTTs (Liu et al., 2016). Our results revealed distinct substrate preferences of CrDGTT1, CrDGTT2, and CrDGTT3 for acylCoAs: CrDGTT1 preferred polyunsaturated acyl-CoAs, CrDGTT2 favored monounsaturated acyl-CoAs, whereas CrDGTT3 preferred C16-CoAs. We were also able to distinguish DGTT preference for prokaryotic DAGs $(s n-2$ being C16) and eukaryotic DAGs (sn-2 being C18): CrDGTT1 preferred prokaryotic DAGs, and CrDGTT2 and CrDGTT3 preferred eukaryotic DAGs (Liu et al., 2016).

Efforts have been make to study DGTT function in vivo through overexpression and knockdown of CrDGTT1, CrDGTT2, or CrDGTT3 (Deng et al., 2012, Iwai et al., 2014, La Russa et al., 2012); however, changes in TAG content and its fatty acid composition were not reported. We employed a microRNA-mediated gene silencing and lipidomic analysis approach to study CrDGTT functions in vivo (Liu et al., 2016). Knockdown of CrDGTT1, CrDGTT2, and CrDGTT3 individually led to different changes in not only TAG content but also fatty acid profiles, consistent with the in vitro assay results. Taking together, we infer that CrDGTT1 is mainly responsible for the incorporation of C18 PUFAs into TAG, CrDGTT2 prefers unsaturated C16 and C18 acyl-CoAs, and CrDGTT3 is active towards C16 saturated and monounsaturated fatty acids as substrates (Liu et al., 2016). Our results suggest the distinct substrate specificities of CrDGTTs may be responsible for the accumulation of diverse TAG species in C. reinhardtii under $\mathrm{N}$ deprivation.

DGAT2 is generally believed to reside in the ER for TAG assembly (Chapman and Ohlrogge, 2012). Unlike higher plant TAG, Chlamydomonas TAG contains mostly (up to 90\%) C16 fatty acids in the $s n-2$ position, suggesting TAG is mostly synthesized through precursors from the plastid via the prokaryotic pathway in $C$. reinhardtii (Fan et al., 2011). Consistently, a prokaryotic pathway has been suggested to be involved in TAG synthesis and lipid droplets (LDs) have been speculated to originate at least in part from the chloroplast of $C$. reinhardtii (Fan et al., 2011, Goodson et al., 2011, Tsai et al., 2015). As a result, DGATs or novel TAG synthesis enzymes involved in prokaryotic TAG synthesis have been predicted (Liu and Benning, 2012). However, prediction programs trained with plant chloroplast sequences failed to predict any plastid targeting sequences in any of the CrDGAT/DGTT enzymes. Our recent in vitro and in vivo DGAT assays revealed that CrDGTT1 preferred prokaryotic DAGs (Liu et al., 2016), suggesting CrDGTT1 is likely involved in prokaryotic TAG synthesis. Subcellular localization determination by immunoblotting indicates that CrDGTT1 probably resides in both the ER and chloroplast envelope (Liu et al., 2016), indicating CrDGTT1 may have access to the outer envelope of the chloroplast and is responsible for prokaryotic TAG synthesis. This work also suggests that unlike plant DGAT2s, CrDGTT1, CrDGTT2, and CrDGTT3 possess distinct specificities towards various acyl-CoAs and DAGs and likely work in concert spatially and temporally to synthesize diverse TAG species in C. reinhardtii (Liu et al., 2016). 
DGAT2s have also been studied in the oleaginous heterokont microalga Nannochloropsis. Although the Nannochloropsis genomes are only one-fourth the size of the $C$. reinhardtii genome, a noticeable enrichment of selected lipid biosynthetic genes are observed in six strains of Nannochloropsis (Wang et al., 2014). In N. oceanica IMET1, under N-deprivation conditions TAG enhancement was attributable to upregulation of seven DGAT genes (Li et al., 2014). IMET1 also exhibited significantly greater relative $D G A T$ transcript abundance under both $\mathrm{N}+$ and $\mathrm{N}$ - conditions compared to $C$. reinhardtii and other non-oleaginous microalgae.

Compared to DGAT2 (or DGTTs), DGAT1 is less studied in these organisms. A Chlamydomonas nitrogen-responsive regulator-1 (CrNRR1) gene, CrNRR1, is suggested to be associated with transcriptional regulation of lipid biosynthetic genes like DGAT1 (Boyle et al., 2012). Knockout of CrNRR1 resulted in 50\% decrease in TAG content in C. reinhardtii under nitrogen deprivation conditions (Boyle et al., 2012).

\subsection{Alternative routes to TAG synthesis}

\subsubsection{Phospholipid:DAG acyltransferases}

Apart from the acyl-CoA dependent Kennedy pathway, an acyl-CoA-independent pathway of TAG synthesis catalyzed by phospholipid:DAG acyltransferases (PDATs) has been characterized in microalgae, which produces TAG by transferring a fatty acyl moiety from a phospholipid (PL) to DAG. A PDAT-encoding gene from $C$. reinhardtii (Cr-PDAT) has been cloned and functionally characterized using biochemical and reverse genetics approaches (Yoon et al., 2012). PDAT is a multifunctional enzyme mediating membrane lipid turnover and TAG synthesis in C. reinhardtii (Yoon et al., 2012). Cr-PDAT transcript knockdown by $65 \%$ with artificial microRNA resulted in a decrease in TAG content by $58 \%$ under N-replete conditions (Yoon et al., 2012), suggesting Cr-PDAT is likely the major contributor of TAG synthesis under N-replete growth conditions. Cr-PDAT utilizes chloroplast membrane lipids, particularly monogalactosyl diacylglycerol (MGDG), sulfoquinovosyl diacylglycerol (SQDG), and phosphatidylglycerol (PG) as substrates to synthesize TAG in vitro. In contrast to At-PDAT, CrPDAT shows high activity with anionic phospholipids such as phosphatidic acid (PA), phosphatidylserine (PS), phosphatidylinositol (PI) and PG. This preferential activity of Cr-PDAT can be partially explained by the fact that PG and PI are predominantly found in the lipidome of C. reinhardtii while cationic phospholipids like phosphatidylcholine (PC) are absent (Yoon et al., 2012). It is speculated that the substrate specificity of PDAT coevolved with the membrane phospholipid composition in various eukaryotes to maintain proper membrane function (Li et al., 2013). Unlike plants which have multiple PDATs, C. reinhardtii contains single Cr-PDAT having lipase activity with broad substrate specificity towards membrane lipids and neutral lipids in vitro. This catalytic promiscuity of Cr-PDAT is carried out by its phosphatidylcholine-sterol O-acyltransferase (LCAT) domain having Ser/Asp-Glu/His triad with lipases, esterases, and proteases activity (Yoon et al., 2012). Although it is unusual for a primary metabolic enzyme to possess multiple catalytic activities, it has been suggested that such catalytic promiscuity might be advantageous for organisms that are challenged by rapidly changing environmental conditions and the constraints of a relatively compact genome (Yoon et al., 2012).

\subsection{Physiological function of TAG synthesis}


Photosynthesis in algae appears to be functionally coupled to TAG biosynthesis and LD biogenesis. Under high-light or $\mathrm{N}$-depleted conditions, excessive electrons generated from photosynthesis result in over-reduction of the photosynthetic electron transport chain and production of cytotoxic reactive oxygen species (Hu et al., 2008). Stress enhanced TAG accumulation may act as an electron sequestration mechanism by scavenging excessive electrons present in the system. Mechanistic insights of this phenomenon were provided through characterization of galactoglycerolipid lipase-encoding gene PGD1 in Chlamydomonas. A pgd1 knockout mutant showed about 50\% decrease in TAG:fatty acids ratio (Li et al., 2012). The pgdl mutant was nonviable when subjected to N-deprivation and can be rescued by blocking the photosynthetic electron transport (Li et al., 2012), suggesting TAG accumulation protects algal cells from oxidative stress.

TAG accumulation is likely a survival strategy of algal cells under stress, and specifically under $\mathrm{N}$ deprivation. In response to $\mathrm{N}$ deprivation, algal cells enter into a cellular quiescence that is characterized by cessation of growth to conserve energy and nutrients. Tsai et al., 2014 provided a mecanistic insight into how TAG plays a critical role in facilitating quiescence exit. These authors identified a gene, compromised hydrolysis of triacylglycerols7(cht7) and its corresponding protein (CHT7), which acts as the repressor of quiescence in C. reinhardtii (Tsai et al., 2014). The cht7 mutant was unable to rapidly degrade TAG following N-resupply and was imparied in the reversal of $\mathrm{N}$-deprivation-induced quiescence. Tsai et al. concluded CHT7 could be a potential target for engineering high TAG accumulating microalge that are not compromised for growth rate.

Under $\mathrm{N}$ deficit, the main constituent of elevated TAG pools in many microalgae (including Nannochloropsis gaditana) consists of saturated and monounsaturated fatty acids (Simionato et al., 2013, Tonon et al., 2002). In N. gaditana, the increase in TAG is proportional to the decline in long-chain polyunsaturated fatty acid (LC-PUFA) membrane lipid content (Simionato et al., 2013). The carbon flux distribution and regulation of TAG pathway under stress is not fully understood in microalgae. It has been observed that $\mathrm{N}$ deficit down-regulates the expression of the enzymes involved in LC-PUFA biosynthesis in Nannochloropsis oceanica IMET1 (Li et al., 2014). It has been proposed that during early $\mathrm{N}$ deficit, membrane lipids are possibly converted to TAG (Jia et al., 2015). Apart from $\mathrm{N}$ deficit, several other stress factors such as $\mathrm{pH}$, temperature, salinity, and phosphorous and carbon limitation also induce TAG accumulation in microalgae (Wase et al., 2015). Labeling experiments in microalgae demonstrated that TAG acts as a reservoir for PUFAs and can be used for rapid formation of plastidic lipids (Cohen et al., 2000). It is hypothesized that upon onset of stress conditions FAs are stored transiently as TAG and subsequently recycled to form plastidic lipids if the stress is mild. In the event of severe stress conditions, TAG would be metabolized to sucrose as part of the adaptation mechanism (Cohen et al., 2000).

Carotenoid biosynthesis may be coupled with TAG synthesis to scavenge excessive electrons or reactive oxygen species ( $\mathrm{Li}$ et al., 2008). In a green alga Haematococcus pluvialis, the majority of fatty acid-esterified astaxanthin molecules are stored in TAG-rich LDs and astaxanthin synthesis consumes about $10 \%$ of molecular oxygen evolved from photosynthesis, reducing the reactive oxygen species production ( $\mathrm{Li}$ et al., 2008). Astaxanthin ester formation is likely catalyzed by DGATs, which have been shown to play an important role in coordinating carotenogenesis and de novo fatty acid biosynthesis in H. pluvialis (Chen et al., 2015). LDs rich 
in carotenoids are generally distributed towards the outer periphery of the Haematococcus cell and are photoprotective for the cell (Hagen et al., 1993).

Clearly much has been learned from the above-described biochemical and molecular genetic analyses, but progress toward understanding microalgal FA and TAG metabolism could be accelerated by expanding computational approaches that help to make sense of the vast amounts of genomic, transcriptomic, proteomic, and metabolomic data that are now available. In the following section we describe these approaches and how they are being (and might be) applied to this problem.

\section{State of the art computational modeling of microalgae}

As computational power has improved (primarily within the last ten years), computational models have begun to be applied for data analysis and pathway prediction in microalgae, enabling rational genetic engineering of algae for biofuels and bioproducts. There are many different types of computational models with varying degrees of quantitative versus qualitative characteristics, each having their own strengths and limitations. Many species have been modeled, although the major focus has been on $C$. reinhardtii since it possesses the first algal genome to be sequenced and it has been the most extensively studied (Bolling and Fiehn, 2005, Choi et al., 2013, Dal'Molin et al., 2011, May et al., 2008, Merchant et al., 2007, Moellering and Benning, 2009, Nguyen et al., 2011, Terashima et al., 2011, Wang et al., 2012).

Several aspects of microalgal behavior have been described, varying from effects of light intensity on photosynthesis to biomass production (Boyle and Morgan, 2009, Chang et al., 2011). Computational models allow predictions to be made about the relationships between microalgal growth, external resources and the products of metabolism with less expense than experiments carried out in a traditional "wet" laboratory. In order to do rational metabolic engineering effectively the metabolic pathways controlling assembly of TAG must be well understood. To facilitate this process, metabolic reconstructions are often created from genome, pathway, and biochemical data found in a variety of databases such as the Kyoto Encyclopedia of Genes and Genomes (KEGG) and MetaCyc, a multiorganism database of metabolic pathways and enzymes (Boyle and Morgan, 2009). Models from Chang et al. (Chang et al., 2011) and Gomes de Oliveira Dal'Molin et al. (Dal'Molin et al., 2011) published in 2011 are complete metabolic reconstructions, with the latter using the terminology GEM for GEnome-scale Metabolic reconstruction.

The major types of models used to describe biochemical systems are topological network or graph analysis, stoichiometric analysis, flux balance analysis (FBA) and kinetic modeling. Some of these models can be applied to entire metabolic networks, which in the case of $C$. reinhardtii and many other microalgae, is the entire organism. One central trait of all these approaches is the need to understand the flow of metabolites through a metabolic pathway. Of these approaches, FBA and kinetic modeling demonstrate the greatest potential to guide efforts to enhance TAG production in microalgae since these are the most quantitative methods. The following equations can be used to describe the basis of kinetic and FBA models (Baroukh et al., 2015a, Edwards et al., 2002, Engel, 2006, Gilbert, 2009, Kauffman et al., 2003).

$$
A+3 B+2 C \rightarrow 4 D
$$




$$
\frac{\mathrm{d}}{\mathrm{dt}}\left(\begin{array}{l}
A \\
B \\
C \\
D
\end{array}\right)=N v=N\left(\begin{array}{l}
v_{A} \\
v_{B} \\
v_{C} \\
v_{D}
\end{array}\right)=0
$$

376

377

378

379

380

381

382

383

384

385

386

387

388

389

390

391

392

393

394

395

396

397

398

399

400

401

402

403

404

405

406

407

408

409

410

411

412

413

414

$$
\begin{gathered}
\text { Reaction Rate }=-\frac{d[A]}{d t}=-\frac{d[B]}{3 d t}=-\frac{d[C]}{2 d t}=\frac{d[D]}{4 d t} . \\
=-v_{A}=-\frac{v_{B}}{3}=-\frac{v_{C}}{2}=\frac{v_{D}}{4}
\end{gathered}
$$

Equation 1 describes the production of $4 \mathrm{D}$ molecules from the reaction of $1 \mathrm{~A}, 3 \mathrm{~B}$, and $2 \mathrm{C}$ molecules. In FBA, Equation 2 applies where the rate of loss or gain of the molecules is related to a stoichiometric matrix of reactants and products, $\mathrm{N}$ and the flux, $\mathrm{v}$, of each of the individual metabolites. These are set to a quasi-steady state, where the incoming and outgoing fluxes must all sum to zero. Thus, the incoming fluxes of the reactants $\mathrm{A}, \mathrm{B}$, and $\mathrm{C}$ must be equal to the product $\mathrm{D}$ outgoing flux in this simple example. One can see how the fluxes are related to the rates of change of concentration for each quantity in Equation 3, where for example the flux associated with loss of $A$ (i.e. the rate at which molecules of A are lost) is equal to $1 / 3$ of the flux of $\mathrm{B}$ and $1 / 4$ of the flux associated with the production of D. FBA and kinetic models differ in applying either equation 2 or 3 a respectively.

We note that computational modeling of microalgae has been reviewed in two recent articles by Baroukh et al. (Baroukh et al., 2015a, Baroukh et al., 2015b). In one article the authors discuss how different models describe the autotrophic, heterotrophic and mixotrophic growth phases of microalgae, while briefly discussing the methods employed in these models (Baroukh et al., 2015a). The other article describes the ways in which the models represent parts of the metabolism, such as photosynthesis, as well as how biomass and macromolecules are represented (Baroukh et al., 2015b). Another review written by Reijnders et al. briefly covers the different models in existence as of 2014 but does not go into detail about them individually, with only brief discussion of the types of models (Reijnders et al., 2014). A review by De Bhowmick et al. discussed methods of metabolic engineering using techniques such as FBA, but did not provide details of how the individual models work (De Bhowmick et al., 2015). While the articles by Baroukh et al. and others are useful for understanding how areas of the metabolism are characterized in all the models that exist to date, it is also beneficial to compare and contrast the various modeling techniques. In particular, the focus of this section is to examine the types of computational models useful for describing FA and TAG production in microalgae, to discuss the pros and cons of the different approaches, and provide examples of how model predictions are tested by experiments.

\subsection{Flux Balance Analysis and lipid biosynthesis}

FBA has been introduced relatively recently (Edwards et al., 2002, Orth et al., 2010). FBA examines the flow of substances through a metabolic system while applying the law of conservation of mass to the reactions in the system. FBA can be applied to entire metabolic networks and assumes steady state conditions, where the flux of each metabolite into the system must equal the flux of that metabolite leaving the system. Metabolic flux analysis (MFA) is a 
variant of FBA where methods such as mass spectrometry are used to determine the levels of metabolites needed to calculate the fluxes required as inputs. With these definitions, we consider FBA to include those approaches that apply equation 2 without using experiments to determine metabolite fluxes whereas MFA approaches do employ experimentally determined fluxes. In a review of different modeling techniques, Nikoloski et al. (Nikoloski et al., 2015) noted that MFA is based on fitting numerical fluxes to experimental data rather than determining analytical solutions to describe the fluxes as is done for FBA. These authors also noted that FBA does not provide a unique steady-state flux distribution and instead flux variability analysis (FVA) must be performed to determine the range of fluxes that meet the optimal objective function.

In either case, boundary conditions are set up for each reaction that takes place in the organism and the flux for every metabolite computed. One can learn which ranges of fluxes are consistent with the boundary conditions provided. In addition, one can specify objective functions that further restrict the possible ranges of flux. For example, one could specify in the FBA that production of biomass or TAG be maximized or that nutrient use be minimized (Raman and Chandra, 2009, Wu et al., 2015). It is worth noting that Kleesen et al. have developed an approach that essentially incorporates elements of both MFA and FBA by integrating FBA with time resolved transcriptomic and metabolomic data (Kleessen et al., 2015).

FBA could be used to study the lipid metabolic pathway and to suggest enzymes with largest effects on lipid production. For example, one could choose an objective function that maximizes lipid production. A sensitivity analysis of the lipid pathway reactions could then be performed, changing the constraints that apply to each one individually to determine the impact on lipid flux. These techniques are most effective if applied to GEMs such as those of Chang et al. (Chang et al., 2011) or Gomes de Oliveira Dal'Molin et al. (Dal'Molin et al., 2011).

GEMs are often employed to incorporate proteome, transcriptome, and metabolome data into FBA models (Dal'Molin et al., 2011). As of 2011, GEMs had been constructed for yeast, mouse, human, and Arabidopsis. One challenge in creating a GEM for algae is the lack of compartmentalization data. However, one example of a GEM for microalgae is the genome scale model AlgaGEM, developed by Gomes de Oliveira Dal'Molin et al. (Dal'Molin et al., 2011). This model describes the autotrophic, mixotrophic, and heterotrophic growth phases and includes the cytoplasm, mitochondrion, plastid, and microbody as separate compartments. The two types of objectives employed for AlgaGEM were minimization of the utilization of energy and carbon sources and maximization of hydrogen gas production. AlgaGEM was used to examine the physiological pathways governing hydrogen production under heterotrophic conditions.

Chang et al. published another GEM in 2011 called iRC1080 (Chang et al., 2011), designed to model light effects on algae in order to maximize absorbed energy and biomass production. One finding of these authors was that the chloroplast was highly central to metabolism, with greater than $30 \%$ of all cell reactions being located in the chloroplast. In modeling metabolism of light utilization, iRC1080 identified effective spectral ranges for light through analysis of reaction activity or absorbance of different light wavelengths. This model was able to predict the number of emitted photons that propel metabolic reactions and simulated autotrophic, heterotrophic, and mixotrophic growth by varying light regulation and environmental exchange constraints. While both GEMs described above were developed with the goal of studying other metabolic processes in microalgae, these models could be used to examine lipid production in these organisms.

Recently, Imam et al. published an updated and improved version of the Chang et al. iRC1080 genome-scale reconstruction from 2011 (Imam et al., 2015). This model incorporated new genes 
and improved reactions, for example adding cofactors that had been missing from the 2011 Chang et al. model. The maximum yield of TAG when using light as an energy source (autotrophic growth) is predicted more accurately by the Imam et al. iCre1355 model. This is an improvement over the model of Chang et al., which gives an estimated TAG yield higher than the theoretical maximum value. Imam et al. suggest that this deficit is due to the reversibility of the pyruvate carboxylase reaction employed in the model of Chang et al. In contrast, this reaction is treated as irreversible in the new model of Imam et al. With this improvement, the model of Imam et al. should be able to more accurately test genetic engineering strategies in silico to determine their effect on TAG yields.

\subsection{Metabolic Flux Analysis}

Similar to FBA, MFA seeks to determine how products are formed and energy is produced (Kliphuis et al., 2012). Most metabolic flux analyses to date have been constructed using metabolite concentrations determined via mass spectrometry. As a case in point, a genome-based metabolic mapping and carbon-13 flux analysis of the microalga Chlorella protothecoides was published by Wu et al. in 2015 (Wu et al., 2015). The genome of this organism was sequenced in 2014 , and this was the first metabolic network reconstruction based on that genome. This species was chosen since it produces a large amount of neutral lipids under nitrogen depleted conditions. In this study, Wu et al. used non-stationary ${ }^{13} \mathrm{C}$ metabolic flux analysis (INST-MFA) rather than the traditional steady state MFA. The advantage of INST-MFA is that dynamic isotopic labeling patterns are generated and intracellular fluxes can be assessed. Liquid chromatography mass spectrometry was used to validate metabolite levels and intracellular fluxes were quantified with kinetic isotope tracing. Reactions were localized to the mitochondria, peroxisome, cytosol, and chloroplast. This information was incorporated into an FBA model that employed an objective function maximizing biomass. The specific growth rate predicted by the model was validated by experiments, including ideal growth rates predicted for autotrophic and heterotrophic growth phases. One notable finding from these studies was that during heterotrophic growth acetyl-CoA usage for the generation of fatty acids was 33-fold higher than during autotrophic growth, pointing to a potential strategy for increasing lipid production by increasing acetyl-CoA pools during heterotrophic growth $(\mathrm{Wu}$ et al., 2015). The authors noted that a strategy to take advantage of this would be to inhibit citrate synthase, the initial enzyme in the tricarboxylic acid cycle, which would allow more acetyl-CoA to go into lipid synthesis.

One application of MFA is to predict genetic changes that will optimize a particular trait, by creating mutants in silico that lead to the desired fluxes or products. Kliphuis et al. introduced a model of $C$. reinhardtii designed to be more abbreviated and less compartmentalized than previous FBA models such as those of Boyle and Morgan or Manichaikul et al. (Boyle and Morgan, 2009, Kliphuis et al., 2012, Manichaikul et al., 2009). To construct the model, experimental studies were conducted by the authors to determine biomass composition, lipid content, carbohydrates, amino acids, and nitrogen content after growth of the microalga in a photobioreactor under red LEDs. This metabolic flux determination applies equation 2 used in FBA where the fluxes are multiplied by the stoichiometric matrix. Since the system of reactions was underdetermined, constraints and objective functions for maximizing biomass yield and maximizing ATP yield were applied. The authors found that low growth rates (leading to low biomass yields) were unfavorable due to high maintenance needs. High light supply was also found to be unfavorable due to light saturation. Therefore, the authors suggest that there must be 
a balance between growth rate and light supply in order to maximize biomass and product yield (Kliphuis et al., 2012). It should be straightforward to apply a similar approach to study the production of lipids in microalgae.

\subsection{Dynamic Reduction of Unbalanced Metabolism Modeling}

In 2014, a new form of modeling called Dynamic Reduction of Unbalanced Metabolism (DRUM) was introduced (Baroukh et al., 2014). DRUM is an extension of FBA. However, it differs from FBA in that it only applies the Quasi Steady State Assumption (QSSA) to some subnetworks where metabolites do not accumulate. However, in other reactions metabolites are allowed to accumulate and behave dynamically. In contrast, no metabolites are allowed to accumulate in FBA. This approach allowed the authors to construct a model in cases where information about the genome was unavailable. The DRUM approach uses the method listed below (Baroukh et al., 2014):

1. Generate the metabolic network of the microorganism under study.

2. Cluster metabolic reactions into sub-networks that are assumed to follow the QSSA.

3. Use elementary modes analysis to reduce each sub-network to a set of macroscopic reactions.

4. Find system of Ordinary Differential Equations (ODEs) from macroscopic reactions kinetics.

Intermediate metabolites do not accumulate in the QSSA so that growth and production of biomass result from complete uptake of the substrates. However in DRUM, QSSA only applies to groups of metabolites in a sub-network and metabolites in the interconnections between subnetworks can accumulate (Baroukh et al., 2014).

The microalga Tisochrysis lutea was used as the model organism for this DRUM study (Baroukh et al., 2014). Unfortunately it was not possible to construct a genome scale metabolic network since this alga has not been sequenced. Instead the metabolic network was constructed by comparing similar features of four algal species with completed metabolic networks, resulting in 157 internal metabolites and 162 reactions. This model replicates experimental data describing lipid and carbohydrate accumulation during the day and consumption at night. The authors found that maximum carbon storage occurs 3 hours before sunset, making this the best time to harvest algae biomass for biofuel production (Baroukh et al., 2014). In principle FBA could be used to separately model day and night steady states. However, FBA cannot be used to combine descriptions of these two conditions in order to model them together as can be done with DRUM since it only operates under one steady state.

As a more advanced variant of FBA, DRUM would also be useful for studying lipid biosynthesis in microalgae. DRUM would allow the FBA techniques discussed earlier to be applied to algae that have not been sequenced and also allow one to assess the impact of varied growth conditions, such as that which occurs during the diurnal cycle, on lipid production. As illustrated above, this information could be used to identify the best time to harvest microalgae in order to maximize lipid yield.

An approach that is quite similar to DRUM was recently described by Knies et al. These authors generated a diurnal FBA model describing Emiliania huxleyi (Knies et al., 2015). In this model, storage metabolites (mannitol and lipid biomass precursors) were allowed to accumulate or be 
consumed throughout the diurnal cycle. Knies et al. found that in this microalga, mannitol was the preferred storage metabolite compared to lipids. This group was able to track the formation of mannitol and lipids, as well as transfer of these metabolites throughout the cell and their usage. This method differs from DRUM with regard to the fact that Knies et al. did not attempt to identify an optimal time for harvest, instead looking at daily biomass concentrations and storage amounts. Knies et al. specifically studied the usage, transfer, and storage of chloroplastic lipids, cytoplasmic lipids, and mannitol. Since this study illuminates how storage metabolites are generated and utilized, a similar approach could be useful when applied to microalgae with a higher propensity for storing lipids than E. huxleyi.

\subsection{Models with Thermodynamic and Energetic Constraints}

A method employed by Cogne et al. examined energetic metabolism in the autotrophic growth phase of eukaryotic microalgae using C. reinhardtii as a model organism (Cogne et al., 2011). This approach uses mass balance constraints similar to FBA and DRUM. Biomass constraints were determined by growing a $C$. reinhardtii strain in a photobioreactor at maximum volumetric biomass growth rates. To add a thermodynamic constraint, the second law of thermodynamics was invoked, such that increasing entropy was applied as a flux constraint for each reaction. An objective-based flux prediction was found by combining the thermodynamic and energetic constraints as well as requiring that net flux be zero. The solution space was dramatically reduced from 90,000 flux distributions to 3,079 when thermodynamic constraints were applied (Cogne et al., 2011). This model illustrated the connections between respiration and photosynthesis in microalgae with changing photon flux (Cogne et al., 2011).

Like traditional FBA and DRUM, this model could apply similar techniques of maximizing lipids as an objective function and sensitivity analysis to understand how changing constraints on different reactions leads to increased lipid production. This approach has the added benefit of incorporating thermodynamic constraints that reduce the solution space.

\subsection{Kinetic Modeling to Enhance Lipid Production}

A kinetic model generally consists of rate equations containing rate constants, initial concentrations, and reaction orders describing the chemical reactions of interest. However, kinetic models can also be constructed that track the time dependent behavior of other variables such as growth rate. The rate equation defines the change in a particular variable with respect to the change in time. For example, if we consider equation 2, the rate law shown below consists of the rate constant for that reaction and the initial concentrations of the three metabolites where $\mathrm{x}$, $\mathrm{y}$, and $\mathrm{z}$ are the experimentally determined orders of the particular metabolite.

$$
\text { Rate }=k_{1}[A]^{x}[B]^{y}[C]^{z}
$$

When multiple reactions are considered, the resulting rate equations become a series of coupled ordinary differential equations (ODEs). An ordinary differential equation solver such as ode15s in MATLAB is used to determine a set of solutions to the ODEs. In a kinetic model of chemical reactions, the inputs to the ODE solver are chemical concentrations of the metabolites as well as the ordinary differential rate equations, and the outputs will be concentrations of those metabolites as a function of time (Ullah et al., 2006). 
Tevatia et al. developed a kinetic model describing autotrophic growth and its relationship to ammonium concentration and neutral lipid accumulation in $C$. reinhardtii since nitrogen depletion is known to induce lipid production in algae (Tevatia et al., 2012). This study varied ammonium concentrations during autotrophic growth while carbon dioxide was the only carbon source (Tevatia et al., 2012). A high throughput analysis was conducted in which fluorescence of Nile red solution was used to quantify neutral lipid levels in the microalgal sample. In their initial experimental studies they found that nitrogen depletion led to increased lipid production, but lower cell growth rate. Kinetic modeling was conducted with the Baranyi-Roberts differential equations for growth and the Luedeking-Piret differential equation for neutral lipid production, fitting the experimental results with these equations to find the equation parameters. It was shown that the model equations predicted cell growth and lipid production matching their earlier experimental results. The authors experimentally found that the nitrogen replete conditions led to little lipid accumulation, with nitrogen-depleted conditions leading to much higher percentages of dry weight lipids and then tailored their model to reflect this. However, the dry weight of cells also decreased. The maximum specific growth rate was found to be dependent on the ammonium concentration in their model. Theoretically, synthesis of lipids became less correlated to growth with increased ammonium ion concentration. The authors concluded that growing algae at varied concentrations of ammonium may lead to higher productions of lipids. (Tevatia et al., 2012)

Two additional models described below also fit under the kinetic modeling classification since they involve rate equations and concentrations as a function of time (Packer et al., 2010, Yang et al., 2010); they define microalgal growth and growth rate in a manner that is very similar to that described by Tevatia et al. by relating the concentration of algae to growth rate. Yang et al. modeled microalgal growth, lipid production and glycerin consumption under photoheterotrophic conditions (where light is needed for use of carbon sources from organic compounds) using Chlorella minutissima as a model organism (Yang et al., 2010). Initially Yang et al. conducted experiments growing $C$. minutissima in photoheterotrophic conditions measuring dry biomass, lipid production, and glycerin consumption parameters for the model. Sensitivity analysis was conducted to determine if observations could be used to estimate parameter values and a calibration model created using Universal Inverse Modeling Code (UCODE) (Yang et al., 2010). The study found that growth and lipid production were most dependent on initial glycerin concentration (Yang et al., 2010). The model fits experimental conditions with correlation coefficients greater than 0.980 for all three components modeled. The biomass was 26 to 36 times as great for the photoheterotrophic conditions as it was for autotrophic conditions. This study was helpful in determining that adding glycerin and using photoheterotrophic growth conditions can lead to higher biomass and lipid concentrations (Yang et al., 2010).

Packer et al. developed a model to describe growth rate and neutral lipid production in the microalga Pseudochlorococcum as well as other microalgae producing high amounts of lipids (Packer et al., 2010). To determine the contribution of nitrogen to biomass and neutral lipid yield, photobioreactors containing a liter of growth medium were subject to constant irradiance experimentally, since in other previous studies nitrogen depletion and high light had led to increased lipids. Their model was constructed to describe the dependence of growth and lipid production on nutrients and light and then compared to experimental data. The authors noted that iron and phosphorous also influence neutral lipid production so that nitrogen starvation may not explain neutral lipid synthesis on its own (Packer et al., 2010). This model was successful at predicting neutral lipid production in most cases using ecological stoichiometry. 
An additional modeling system by Chang et al. (Chang et al., 2016) was able to determine the relationship between light intensity, dissolved inorganic carbon, maximum biomass concentration, and maximum specific growth rate. In this study the Verhulst logistic kinetic model was employed to look at microalgal biomass concentration over time. The modeling results were validated by performing additional experimental studies. In the future it would be interesting to see if this approach can be extended to describe lipid production.

\subsection{Relevance of computational models to lipid biosynthesis}

It has not been long since computational models of microalgae first came into existence, but much insight has been gained into the relationships between the three growth phases of microalgae, nutrient balances, the effects of light, and biomass production. These models have suggested ways to optimize microalgal growth and predicted genetic changes to improve growth and production of lipids. Attempts by some authors have been successful at modeling growth (Packer et al., 2010, Tevatia et al., 2012, Yang et al., 2010), another study was able to predict lipid accumulation during the day and night cycles (Baroukh et al., 2014), and others helped identify the differences in fluxes between the growth phases (Boyle and Morgan, 2009, Chang et al., 2011, Dal'Molin et al., 2011).

Although these models may not all have been directly applied to enhance lipid production in vivo, one can envision how they could be used for this purpose. The 2011 FBA model of Chang et al. or AlgaGEM could be employed with an objective function designed to optimize lipid production (Chang et al., 2011, Dal'Molin et al., 2011). The model described in 2015 by Imam et al. could also be used with an objective function that maximizes lipid production (Imam et al., 2015). Using INST-MFA combined with FBA, Wu et al. were able to determine that increasing acetyl-CoA pools during heterotrophic growth improves lipid production (Wu et al., 2015) and a similar approach could be applied to other algal growth phases. In another MFA study, Kliphuis et al. suggested that the balance between growth rate and light supply must be carefully adjusted in order to maximize biomass and product yield (Kliphuis et al., 2012). Thus, future studies could attempt to identify the appropriate balance required to maximize lipid production. The DRUM model of Baroukh et al. was able to identify an optimal time to harvest algae for maximal carbon storage (Baroukh, Munoz-Tamayo, 2014). This method could be used to determine conditions that specifically support maximal storage of lipids. The model of Knies et al. examined the role of diurnal effects in modulating transfer between chloroplastic lipids, cytoplasmic lipids, and mannitol (Knies et al., 2015). It should be possible to use this approach to optimize transfer of polar lipids into forms that can be used for biofuels. The FBA model with thermodynamic constraints developed by Cogne et al. (Cogne et al., 2011) could be used with an objective function that maximizes lipids in a similar manner to other FBA models. Finally, as the kinetic models of Tevatia et al., Yang et al, and Packer et al. (Packer et al., 2010, Tevatia et al., 2012, Yang et al., 2010) were able to successfully describe the impact of various experimental conditions on lipid production, it may be possible to identify conditions that stimulate lipid production by optimizing the parameters found in these models. Thus, given the financial constraints and time restrictions that often apply to experimental studies, computational models are an important and useful tool to better understand microalgal metabolism.

In the future, it is likely that more models integrating kinetic parameters into FBA models like DRUM and the approach of Cogne et al. (Cogne et al., 2011) will become more prevalent. These hybrid kinetic and FBA approaches will likely be the models of choice until all the necessary 
kinetic parameters are known and complete kinetic models of the metabolism can be created. We believe that models based on FBA also have great potential to be expanded from QSSA in order to describe the dynamics of metabolic pathways. So-called dynamic FBA allows one to track the time dependent behavior of fluxes and has been performed for several systems including $E$. coli (Barreto-Rodriguez et al., 2014, Grafahrend-Belau et al., 2013, Hohenschuh et al., 2015, Mahadevan et al., 2002). By looking at fluxes over time, the models become much more similar to the more quantitative kinetic models (Table 1), without the need for kinetic parameters. There is much to be learned from computational models of microalgae, which will enable TAG metabolic pathways to be more clearly understood, and facilitate strategies to optimize microalgal growth and the production of desirable substances such as lipids. In this way, the widespread use of these organisms to produce useful fuels will move a step closer to becoming reality.

\section{(Place for Table 1)}

\section{Technical advances that facilitate metabolic engineering}

As more and more studies integrate experiments with mathematical modeling of FA and TAG metabolism, our understanding of these molecules and how to manipulate their production pathways will improve. In this section we review some of the tools that are (or may soon be) useful in engineering microalgal genomes, in ways that facilitate the testing of model predictions and that should enhance TAG production.

\subsection{Transforming microalgae}

Metabolic engineering of microalgae requires developing transformation techniques for the species of interest. Many eukaryotic microalgae nuclear genomes have been transformed (Scaife et al., 2015), although only a modest number of species have been transformed and replicated by independent groups. Examples include the green algae C. reinhardtii, Volvox carteri, Chlorella ellipsoidea, Chlorella vulgaris, Dunaliella salina, and $H$. pluvialis; the heterokonts Phaeodactylum tricornutum, Thalassiosira pseudonana, N. gaditana and N. oceanica; and the red alga Cyanidioschyzon merolae. Several of these species are industrially relevant. In contrast, organellar genomes of microalgae have proven to be much more difficult to transform. Many groups have transformed the chloroplast and mitochondria of $C$. reinhardtii since 1988 (Boynton et al., 1988, Rasala et al., 2010), but there have been only a few reports of successful organellar transformation for other species. A nearly comprehensive list of microalgae that have been transformed to date can be found in the supplemental information of Scaife et al. (Scaife et al., 2015).

A comparison of different transformation techniques used in microalgae is summarized in Table 2 .

\section{(Place for Table 2)}

\subsection{Other technical advances}

C. reinhardtii is by far the best-studied microalga, and seemingly every molecular technique that is in widespread use for major animal, plant, and fungal model systems has been developed for 
766

this species. These include methods for forward genetics (generating mutants through random insertional mutagenesis and then cloning flanking gene sequences associated with the mutant phenotypes), reverse genetic analyses (generating targeted mutants via RNAi), and characterization of protein localization and abundance (via in vivo fluorescent and luminescent reporters). An excellent recent review describes many of these tools and their uses comprehensively (Jinkerson and Jonikas, 2015), so advances in Chlamydomonas methods highlighted by that review will not be described in detail here. Instead this section will focus on advances achieved for various algae in heterologous protein expression and in genome editing, the latter a methodology that is superseding previous reverse genetic approaches and other methodologies and that is revolutionizing genome engineering.

\subsubsection{Heterologous protein expression}

Historically it has been difficult to express heterologous nuclear genes in certain microalgae, due to mechanisms both dependent on and independent of methylation of the transgenic DNA (Babinger et al., 2001, Cerutti et al., 1997). As described in Jinkerson and Jonikas, strategies including codon optimization, inclusion of introns, and use of mutant host strains selected for elevated protein expression have improved the situation, but each of these strategies has limitations. However, recent reports demonstrate that at least $C$. reinhardtii can be reliably engineered to produce heterologous proteins at high levels when they are co-transcriptionally expressed with a selectable marker gene using a viral $2 \mathrm{~A}$ peptide linker sequence. The $2 \mathrm{~A}$ peptide sequence is found naturally in several lentiviral genomes and facilitates ribosome skipping from one ORF to the next (Donnelly et al., 2001); many groups have reported successful co-expression of two or more unrelated genes in other systems using the $2 \mathrm{~A}$ peptide method (reviewed in (Luke et al., 2010)). As long as the 2A peptide sequence ( $20-40$ amino acids) does not hinder function of the first protein expressed from the bi-cistronic gene, the method has the potential to work routinely. Two groups independently reported robust expression of heterologous proteins in Chlamydomonas, using nuclear transgenes that connected bleomycin resistance gene (ble) coding sequence to the gene of interest via FMDV (foot and mouth disease virus) 2A peptide sequence (Plucinak et al., 2015, Rasala et al., 2012). Rasala et al. co-expressed a xylanase from the fungus Trichoderma reesei with the ble protein via a 24-aa 2A peptide linker; xylanase activity was 100 -fold higher in transformants expressing this fusion than in those expressing xylanase the traditional way. In similar fashion, Plucinak et al. reported $>9$-fold increase in the rate of rescue of an FKB12 mutant (which makes Chlamydomonas resistant to rapamycin) when the FKB12 gene product was co-expressed with ble protein via a longer (39-aa) 2A peptide linker, versus adjacent expression (ble and FKB12 expressed from different gene cassettes on the same plasmid). Plucinak et al. tested 5 additional selectable marker genes in their system: AphVIII, paromomycin resistance; AphVII, hygromycin resistance, aadA, spectinomycin resistance; $A R G 7$, arginino succinate lyase, arginine auxotrophy for $\arg 7$ mutants; RBCS2 (Rubisco small subunit 2), for rescue of rbcs mutants. Interestingly, none of these functioned nearly as well as the gene for ble. Kong et al. also recently reported successful high-level expression of a heterologous protein (Botryococcus braunii squalene synthase-like protein) in Chlamydomonas when it was linked to ble by 2A peptide (Kong et al., 2015). It seems likely that the $2 \mathrm{~A}$ peptide strategy should work for other algae, but what is less clear is whether a variation of this strategy might work to express more than two proteins from the same gene regulatory cassette, in order to simplify the multiplexing of heterologous protein expression.

\subsubsection{Genome editing}


Genome editing is fast becoming routine in many organisms (Doudna and Charpentier, 2014, Hilton and Gersbach, 2015), but unfortunately adaptation of this methodology to microalgae has been slow. The general strategy of genome editing is to target DNA double-strand breaks (DSBs) to specific regions of the genome. A DSB is usually repaired imperfectly by non-homologous end joining (NHEJ), introducing a short insertion or deletion into the region, but homologous recombination (HR) can occur if a DNA fragment is provided that is homologous to the targeted region (Naldini, 2011). Therefore a gene of interest can be targeted for mutation. It can also be precisely edited to a different sequence of choice (for instance, a reporter gene can be precisely spliced in). Three major kinds of technologies have been developed for genome editing of organisms for which homologous recombination is not normally a high efficiency event: zincfinger nucleases (ZFNs), transcription activator-like effector nucleases (TALENs), and clustered regularly-interspaced short palindromic repeats (CRISPR). Modified versions of enzymes used in these technologies can also be used to target genes for transcriptional activation or repression (Gaj et al., 2013).

ZFNs combine a bacterial type IIS restriction enzyme DNA-cleaving domain (typically from the enzyme FokI) with arrays of zinc-finger domains (each designed to bind one of the four DNA nucleotides) to recognize specific sequences and to target them for cleavage (Carroll, 2011). A ZFN was recently used to create targeted mutations in the $C$. reinhardtii channelrhodopsin gene (COP3), via a strategy that involved screening among transformants selected for repair of a selectable marker gene into which had been inserted the target site for COP3 mutagenesis; the idea was that some of the cells with repaired marker gene would also contain edits in the endogenous COP3 gene (Sizova et al., 2013). ZFN methodology is somewhat laborious because of the requirement to engineer a specific ZFN protein for each target and targeting is sometimes poor with respect to efficiency and specificity; in the case of this study, identifying 4 COP3 mutants required screening more than 100 transformants by PCR. However, this report provided the first evidence that genome editing can work in Chlamydomonas.

The TALENs strategy was developed subsequently, and like ZFNs, TALENs combine a DNAbinding domain (in this case it is called the TALE domain) with the FokI nuclease domain (Boettcher and McManus, 2015). The TALE domain is constructed by arraying sets of 34-amino acid repeats, each containing a repeat variable diresidue (RVD) occurring at amino acid positions 12 and 13 within the repeat. The other 32 amino acid positions are constant. Each of four different diresidues- $\mathrm{HD}, \mathrm{NI}, \mathrm{NG}$, and $\mathrm{NN}$ - specifies binding at a specific nucleotide, namely $\mathrm{C}, \mathrm{A}, \mathrm{T}$, and $\mathrm{G}$, respectively. TALENS have been used to create both NHEJ mutations and to do high-efficiency HR in the diatom $P$. tricornutum. NHEJ mutations in a targeted gene were created at a rate of $>25 \%$ of transformants that expressed the TALEN (Daboussi et al., 2014), while HR events occurred at a rate of $24 \%$ (Weyman et al., 2015). Thus, TALENs appear to be a very efficient tool for genome editing in diatoms and presumably could be a successful strategy for generating precise mutations and gene fusions in other algae. In fact, they have already been used to increase expression of the two arylsulfatase genes, $A R S 1$ and $A R S 2$, in $C$. reinhardtii (Gao, 2014). The biggest drawback to TALENs is that, as is the case for ZFNs, a new TALEN must be designed and generated for each targeted mutation, so implementing this methodology can be laborious and expensive.

Arguably CRISPR is the easiest and most efficient genome editing technology, and involves the use of a Cas9 endonuclease (Jinek et al., 2012). Cas9 enzymes contain endonuclease domains that cleave opposite strands of a DNA sequence and can be targeted to specific sequences by a 
guide RNA that contains a region perfectly homologous to that sequence. The advantage of CRISPR/Cas9 over ZFNs and TALENs is that a single enzyme can be targeted to many different sequences so there is no need to generate a new enzyme for every new sequence targeted. A minor disadvantage of the CRISPR technology is that each targeted NHEJ mutation requires two components (Cas9 and a specific guide RNA) to be provided for an editing event, and HR also requires a DNA template for repair. So far no practical successes have been reported for CRISPR/Cas9 in algae, though one mutant was generated via this system in C. reinhardtii (Jiang et al., 2014). Constitutive Cas9 expression appears to be toxic in this alga but hopefully future vectors featuring either an inducible Cas9 gene or alternative delivery of Cas9 protein and sgRNA (such as by electroporation) will overcome this problem.

Advances in transformation and other techniques for genome modification will eventually allow targeted modification of algal genomes, whereas molecular, biochemical, and computational model analysis will inform how best to modify these genomes for enhanced lipid production and predict the effects of such changes. Below we provide an overview of reported metabolic engineering attempts aimed to improve traits for TAG and biomass production in algae, and we provide suggestions for future efforts.

\section{Engineering algae for enhanced TAG production}

\subsection{Theoretical maximum yield of TAG}

Before discussing various metabolic engineering strategies for enhanced TAG production, it is useful to consider the upper limit of any improvements: what is the theoretical maximum yield of TAG that can be obtained from photosynthetic microalgae? Below, we calculate the theoretical maximum yield of TAG per mol of photons to establish the magnitude of improvement for TAG production that is possible in principle. The approach taken is similar to that described by Johnson and Alric in their recent review (Johnson and Alric, 2013), where assumptions were made that algae were grown under conditions optimized for TAG production. Thus, photons needed to make proteins and carbohydrates are minimal while photoinhibition is negligible. The information provided in Table 2 of that work can be employed to determine the amount of energy (ATP, NADPH, and $\mathrm{NAD}^{+}$) required to make different components of fatty acids. For example, the production of a C16 fatty acid, palmitic acid, entails a cost of 8 acetyl-CoA, 7 ATP, $14 \mathrm{NADPH}$, and $16 \mathrm{NAD}^{+}$while one molecule of acetyl-CoA requires $7 \mathrm{ATP}$ and $6 \mathrm{NADPH}$. Breuer et al. (Breuer et al., 2014) noted that the energy requirements for generating the glycerol backbone are 7 NADPH and 9 ATP, with 1 ATP used for each condensation of acid to the backbone. As there are three acid condensation reactions to form a TAG molecule, the total utilization of energetic cofactors is $7 \mathrm{NADPH}$ and 12 ATP per glycerol molecule during TAG formation. To calculate the total energy requirements for generating TAG containing palmitic acid, one multiplies the energetic cost of producing palmitic acid by 3 and adds the requirements for the glycerol backbone as follows:

8 acetyl-CoA $=56 \mathrm{ATP}+48 \mathrm{NADPH}$

1 palmitic acid chain

$=8$ acetyl-CoA $+7 \mathrm{ATP}+14 \mathrm{NADPH}+16 \mathrm{NAD}^{+}$

$=63 \mathrm{ATP}+62 \mathrm{NADPH}+16 \mathrm{NAD}^{+}$ 
870

871

872

873

874

875

876

877

878

879

880

881

882

883

884

885

886

887

888

889

890

891

892

893

894

Combining 3 acid chains to generate each TAG molecule leads to: 189 ATP +186 NADPH +48 $\mathrm{NAD}^{+}$

Adding the requirements for condensing acid chains to the glycerol backbone per TAG molecule gives: $201 \mathrm{ATP}+193 \mathrm{NADPH}+48 \mathrm{NAD}^{+}$

One can make the assumption suggested by Breuer et al. (and adopted by Imam et al.) (Breuer et al., 2014, Imam et al., 2015) that all the NADH produced from $\mathrm{NAD}^{+}$in this process is used to reduce $\mathrm{NADP}^{+}$to NADPH. In this case, $48 \mathrm{NADPH}$ molecules would be regenerated and the net totals are:

201 ATP + 145 NADPH

With this assumption the balanced reaction to produce one TAG molecule $(\mathrm{C} 16: 0)$ is:

$51 \mathrm{CO}_{2}+201 \mathrm{ATP}+145 \mathrm{NADPH}+145 \mathrm{H}^{+} \rightarrow \mathrm{C}_{51} \mathrm{H}_{98} \mathrm{O}_{6}[\mathrm{TAG}(\mathrm{C} 16: 0)]+96 \mathrm{H}_{2} \mathrm{O}+145$ $\mathrm{NADP}^{+}+201 \mathrm{ADP}+201 \mathrm{Pi}$

Conversely, if this assumption is not made:

$51 \mathrm{CO}_{2}+201 \mathrm{ATP}+193 \mathrm{NADPH}+48 \mathrm{NAD}^{+}+145 \mathrm{H}^{+} \rightarrow \mathrm{C}_{51} \mathrm{H}_{98} \mathrm{O}_{6}[\mathrm{TAG}(\mathrm{C} 16: 0)]+96 \mathrm{H}_{2} \mathrm{O}$ $+193 \mathrm{NADP}++201 \mathrm{ADP}+201 \mathrm{Pi}+48 \mathrm{NADH}$

Breuer et al. computed the mass of TAG per mol photons by noting that 8 photons yield 3 ATP and 2 NADPH via linear electron transport in photosynthesis (Breuer et al., 2014). The molar mass of TAG with palmitic acid (palmitin) is $807 \mathrm{~g}$. Taking the number of NADPH molecules to be 145 or 193 in the balanced reactions above (depending on whether one assumes the regeneration of NADPH from NADH), dividing by 2 and multiplying by 8 gives the moles of photons required for production of a TAG (C16:0) molecule in the balanced reaction, in this case 580 or $772 \mathrm{~mol}$ photon respectively. To determine the mass of TAG/mol photon the molar mass of TAG is divided by the mol of photons, which gives a theoretical maximum yield of $1.39 \mathrm{~g}$ $\mathrm{TAG} / \mathrm{mol}$ photon when the assumption of NADPH regeneration is made or $1.05 \mathrm{~g} \mathrm{TAG} / \mathrm{mol}$ photon when that is not done.

Breuer et al. (Breuer et al., 2014) calculated the energy requirements for triolein and find the balanced reaction to be:

$57 \mathrm{CO}_{2}+217 \mathrm{NADPH}+225 \mathrm{ATP}+54 \mathrm{NAD}^{+}+157 \mathrm{H}^{+} \rightarrow \mathrm{C}_{57} \mathrm{H}_{104} \mathrm{O}_{6}[\mathrm{TAG}(\mathrm{C} 18: 1)]+108 \mathrm{H}_{2} \mathrm{O}$ $+217 \mathrm{NADP}++54 \mathrm{NADH}+225 \mathrm{ADP}+225 \mathrm{Pi}$

Breuer et al calculated the theoretical yield to be 868 mol photons required for 1 mol of triolein which equals $1.02 \mathrm{~g}$ TAG/mol photon. These authors note that the yield can be increased to 1.36 $\mathrm{g}$ TAG/mol photon if the assumption is made that NADH reduces NADP+ to NADPH. 
Imam et al. did a similar calculation of the theoretical maximum yield for a TAG species found to be most abundant in $C$. reinhardtii. This species contains a saturated palmitic acid, an oleic acid with one point of unsaturation, and a saturated oleic acid. The resulting balanced energy equation is:

$55 \mathrm{CO}_{2}+156 \mathrm{NADPH}+156 \mathrm{H}++208 \mathrm{ATP} \rightarrow \mathrm{C}_{55} \mathrm{H}_{104} \mathrm{O}_{6}(\mathrm{TAG} \mathrm{C16:0,} \mathrm{C18:1,} \mathrm{C18:0)}+104$ $\mathrm{H}_{2} \mathrm{O}+156 \mathrm{NADP}^{+}+208 \mathrm{ADP}+208 \mathrm{Pi}$

As noted above, this reaction assumes that any NADH produced is used to reduce $\mathrm{NADP}^{+}$to $\mathrm{NADPH}$, decreasing the amount of NADPH expended. This calculation leads to a theoretical maximum yield of $1.38 \mathrm{~g}$ TAG/mol.

These calculations allow one to understand the theoretical maximum efficiency of TAG production. It is useful to compare the theoretically possible TAG/mol photon ratios shown above to ratios representing realistic scenarios in order to understand the degree to which existing yields can be further optimized. In order to perform this calculation we made the following general assumptions (Benemann, 2013, Hu et al., 2008):

- A typical open photobioreactor system (e.g., raceway ponds) can produce algal biomass at a rate of up to $15-20 \mathrm{~g} / \mathrm{m}^{2} /$ day.

- The TAG content of the biomass in this system is about $20 \%$.

- Photons are available from sunlight with a Photosynthetic Photon Flux Density (PPFD, which accounts for the $45 \%$ of total flux of solar photons that are available for photosynthesis) of $2000 \mu \mathrm{mol} \mathrm{m} \mathrm{s}^{-2}$ and each day includes 10 hours of sunlight (at an ideal location).

- Photoinhibition (i.e. light induced reduction in photosynthetic efficiency) is not taken into account.

- $22 \%$ of the energy in the photon captured by photosynthesis is used to produce algae biomass.

$\frac{2000 \times 10^{-6} \mathrm{~mol} \text { photons }}{\mathrm{m}^{2} / \mathrm{s}} * \frac{3600 \mathrm{~s}}{\text { hour }} * \frac{10 \text { hours }}{\text { day }} * 22 \%$ sunlight for biomass $=\frac{15.84 \mathrm{~mol} \text { photons }}{\mathrm{m}^{2} / \text { day }}$

$\underline{15-20 \mathrm{~g} \text { biomass }} * 20 \% \mathrm{TAG}$ content $=\underline{3-4 \mathrm{~g} \mathrm{TAG}} \quad / \quad \underline{15.84 \mathrm{~mol} \text { photons }}=0.19-0.25 \mathrm{~g}$

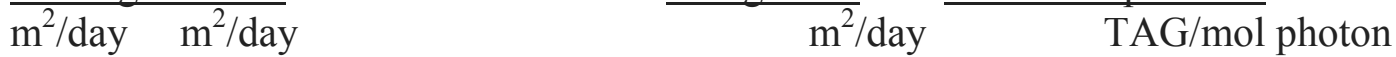

Thus, we estimate that a typical rate of TAG production in an open bioreactor at the present is about $0.19-0.25 \mathrm{~g} \mathrm{TAG} / \mathrm{mol}$ photon, in line with the value obtained by Breuer et al. (Breuer et al., 2014). This is about one fourth of the theoretical maximum yield according to our calculations performed above and reveals that there is substantial room for improvement in optimizing TAG production in realistic systems. Despite the possibility for improvements, it is worth mentioning that it is likely not possible to reach the theoretical maximum yield of $1.38 \mathrm{~g}$ $\mathrm{TAG} / \mathrm{mol}$ photon due to the inevitable loss of energy via photoinhibition and partitioning of some energy into carbohydrate and protein biosynthesis to maintain basic cellular metabolism. 


\subsection{Genetic manipulations aimed at enhancing FA and TAG}

939 Five main engineering strategies have been attempted for enhancing FA and TAG synthesis 940 (quantity and quality) in plants and yeast: (1) Increasing FA precursors, including G-3-P; (2) 941 Inhibition of $\beta$-oxidation of FAs in the peroxisomes or reducing lipase hydrolysis; (3) 942 Overexpression of FA and TAG biosynthetic enzymes; (4) Regulation of thioesterase (TE) for (Hoffmann et al., 2008). Similar strategies have been tested in microalgae, with varying degrees of success. As described below and summarized in Table 3, manipulation of FA biosynthesis genes has not successfully enhanced TAG production; by contrast overexpression of genes in the Kennedy pathway and some transcriptional regulators increased TAG yield significantly, whereas manipulating TE, acyl-ACP desaturase (FAB), and other regulatory genes altered FA composition of TAG.

\subsubsection{FA biosynthetic and the Kennedy pathway genes}

Among FA biosynthesis genes, acetyl-CoA carboxylase1 (ACC1) and beta-ketoacyl-ACP synthase II (Kas II) have been manipulated independently in efforts to increase lipid content by supplying more precursors toward Acyl-ACP. Interestingly, though ACCase catalyzes the first step in FA biosynthesis and has been shown to be a key control point in other organisms, including E. coli and potato (James and Cronan, 2004, Klaus et al., 2004) overexpression of native ACCase did not enhance oil yield in the diatoms Cyclotella cryptica and Navicula saprophila (Dunahay et al., 1996, Radakovits et al., 2010). Similarly, ACCase activity and TAG accumulation have been found to be uncorrelated for Chlorella vulgaris and Chlorella sorokiniana cultured under various growth conditions (Leyva et al., 2015, Wan et al., 2011). These results suggest that ACCase may not be a control point for FA/TAG production in at least some green algae and heterokonts, so overexpression of ACCase might not be a productive strategy for increasing TAG in microalgae. By contrast, overexpressing Kas II, which acts downstream of Accl in the FA biosynthetic pathway, enhanced C18 composition in $C$. reinhardtii but it was not clear from this study whether this manipulation altered the overall quantity of TAG produced (Blatti et al., 2013).

Fatty acid synthases (FAS, EC 2.3.1.85) are multi-functional, multi-subunit enzymatic complexes in which the subunits are functionally interdependent. Under nitrogen deprivation a modest increase in transcription of FAS complex genes, including those encoding ACP and acylACP thioesterase 1 (FAT1), was observed in C. reinhardtii with enhanced accumulation of TAG (Miller et al., 2010), suggesting that ACP and FAT might be targets for improving lipid producing potential. However, reminiscent of the situation for ACCase, initial efforts to enhance FA biosynthesis by introducing plant FAS enzymes into microalgae were not successful (Blatti et al., 2013). Further characterization of algal FAS is required to guide future strategies for metabolic engineering of this enzyme. It is also possible that altering the expression of multiple FA genes or controlling the expression of master regulator(s) operating on the global FA biosynthetic pathway may have more extensive impacts on lipid production (Ohlrogge and Jaworski, 1997).

In contrast to the above-described strategies, the Kennedy pathway manipulations have been fairly successful, especially those involving DGAT overexpression (Table 3). The most significant DGAT-induced increase (20-44\%) in neutral lipid was achieved by overexpressing 
native DGAT2s in P. tricornutum and C. reinhardtii (Deng et al., 2012, Niu et al., 2013). In order to manipulate the entire Kennedy pathway five gene cassettes (G3PDH, GPAT, LPAT, $P A P$ and DGAT from yeast) were used to transform Chlorella minutissima. This strategy increased TAG content by two-fold (Hsieh et al., 2012). Interestingly, channelizing the carbon precursor (Acyl-ACP) towards TAG by overexpression of a Lobosphaera incisa GPAT in $C$. reinhardtii led to $50 \%$ increase in TAG content without compromising growth (Iskandarov et al., 2015), indicating this is a promising strategy for enhanced TAG production. In aggregate, these results generally indicate that the Kennedy pathway genes exert a larger degree of regulation on TAG biosynthesis than FA synthetic genes, suggesting that manipulations that "pull" substrates through the TAG biosynthesis pathway are more effective at increasing TAG production than those that "push" substrates into it.

\section{(Place for Table 3)}

\subsubsection{Substrate specificity and desaturase genes}

Substrate specificity of an acyl-ACP thioesterase is crucial for controlling the carbon chain length and properties of the final fatty alkane product. For example, FatB thioesterases from plants were introduced into the diatom $P$. tricornutum in an attempt to improve its biodiesel feedstock quality. This approach led to a $6.2 \%$ increase in C12:0 and 15\% increase in C14:0 FAs by weight (Radakovits et al., 2011). Overexpression of native $C$. reinhardtii $C r T E$ in the chloroplast resulted in a short-circuiting of fatty acid production and enhanced myristic acid (14:0) accumulation (Blatti et al., 2012). Such medium chain saturated FAs (C8- C14) are best suited for biodiesel production, as shorter FA chains do not generate sufficient energy and longer chain FAs are too waxy compared to current diesel fuels. The length of the fatty acyl chain can also be controlled by introducing chain-length specific acyl-ACP thioesterases via protein engineering (Yuan et al., 1995).

Desaturation of FAs is catalyzed by the enzyme fatty acid desaturase (FAD) which converts C-C single bonds to $\mathrm{C}=\mathrm{C}$ double bonds at specific positions of the fatty acyl chain (Li-Beisson et al., 2015). In green algae, the soluble stromal enzyme stearoyl-ACP desaturase (SAD) determines the ratio of saturated to unsaturated fatty acids and catalyzes the first desaturation reaction converting 18:0-ACP to 18:1 $\Delta^{9}$-ACP. The native SAD has been overexpressed in Chlamydomonas resulting in $>28 \%$ increase in FAs due to a 2.4-fold increase in oleic acid (18:1) along with enhanced production of linoleic acid (18:2) and palmitic acid (16:0) (Hwangbo et al., 2014). It is expected that increased functional validation of genes responsible for algal lipid desaturation will further facilitate rational engineering of FAs with desired states of unsaturation. A more detailed discussion of regulation of fatty acid chain elongation and desaturation is beyond the scope of this review.

\subsubsection{Transcription factor genes and other regulators}

To date, most targets for genetic manipulation of algae have been primarily informed by molecular and biochemical studies. More recently, however, rapid advances in DNA/RNA sequencing and bioinformatics in microalgae have allowed lipid metabolic pathway studies at the whole genome and transcriptome level. These in turn have permitted identification of transcriptional regulators and regulatory proteins of TAG/FA biosynthetic pathway, which have been used to re-engineer cellular lipid content. For example, overexpression of a DOF-type transcription factor increased total lipid production in $C$. reinhardtii by two-fold (Ibanez-Salazar et al., 2014). By contrast, knockout of CrNRR1, a specific TAG regulator for nitrogen 
deprivation, resulted in $50 \%$ decrease in $\mathrm{TAG}$ content in $C$. reinhardtii under nitrogen deprivation conditions (Boyle et al., 2012). A recent whole genome and transcriptome study reconstructed the central carbon metabolism of Chlorella pyrenoidosa, including lipid biosynthesis pathways, and suggested NAD $(\mathrm{H})$ kinase3 (NADK3) controlled NADPH supply and contributed to lipid production in this alga (Fan et al., 2015). Overexpression of an Arabidopsis $\mathrm{NAD}(\mathrm{H})$ kinase in C. pyrenoidosa enhanced cellular lipid content effectively by $110.4 \%$ without slowing down growth rate (Fan et al., 2015), representing a significant increase in lipid production in industrial-relevant algal strains. These studies confirm the potential of omics studies and pathway modeling in identification of metabolic switches for maximizing TAG production in algae.

\subsubsection{Other genes}

Apart from the above examples of TAG/FA biosynthetic and TAG-pathway regulatory genes, some manipulations of genes involved in TAG accumulation and LD formation, photosynthetic genes (PEPC1), and structural genes of the citric acid cycle (citrate synthase, CIS) have demonstrated significant impacts on carbon flux towards TAG biosynthesis (Table 3). For example, overexpression of the lipid-body associated gene PtPNPLA3 (patatin-like phospholipase domain-containing protein 3) enhanced lipid productivity by $70 \%$ in $P$. tricornutum (Wang et al., 2015). Meanwhile, downregulation of PEPCl or CIS in C. reinhardtii increased TAG production by $20 \%$ and $169.5 \%$, respectively (Deng et al., 2013, Deng et al., 2014). Interestingly, a recent and independent genome-based metabolic mapping and FBA of the microalga Chlorella protothecoides predicted inhibition of CIS should lead to redirection of carbon flux into lipid biosynthesis, and thus should be a promising strategy for enhanced TAG production (Wu et al., 2015). This was an example of experimental validation preceding model prediction, but nevertheless demonstrates that modeling of TAG metabolism can be used to accurately predict outcomes of genetic manipulations.

\subsection{Other metabolic engineering strategies and trade-offs}

\subsubsection{Enhancing TAG production through improving biomass yield}

At present, the general consensus of the algal research community is that biomass productivity is a key bottleneck for biofuel production (DOE, 2010). As such, several genetic engineering strategies have been applied to increase algal photosynthetic efficiency and biomass productivity. For instance, reducing antenna size in the photosynthetic machinery to improve light use efficiency under mass microalgal culture has been suggested. Green microalgae such as Chlamydomonas and Haematococcus have naturally small chlorophyll (Chl) antenna sizes which are comparable to those in $\mathrm{C}_{3}$ plants (Ort and Melis, 2011). An insertional mutation that reduced the light-harvesting $\mathrm{Chl}$ antennae even more in $C$. reinhardtii decreased photodamage and increased $P \max$ in this alga (Polle et al., 2003). However, strains with reduced antenna size did not achieve higher biomass productivity under mass culture conditions due to reduced fitness under high light intensity (de Mooij et al., 2015). Therefore, for algal strain engineering it is important to consider greater tolerance to high light and other changing environmental factors outdoors such as temperature and $\mathrm{pH}$.

Another potential strategy for increasing algal biomass is to enhance flux through the Calvin cycle by increasing expression of key Calvin cycle enzymes. This idea seems especially promising, since in multiple plants, overexpression of either (or both) fructose 1,6- 
bisphosphatase (FBPase) or sedoheptulose 1,7-bisphosphatase (SBPase) increases growth and biomass (Feng et al., 2007, Ichikawa et al., 2010, Miyagawa et al., 2001, Tamoi et al., 2006). Interestingly, however, four-fold overexpression of FBPase protein in $C$. reinhardtii, which led to a 1.4-fold increase in FBPase enzyme activity, actually had a significant negative effect on growth, suggesting that unlike the situation in plants, FBPase does not catalyze a rate-limiting step in green algae (Dejtisakdi and Miller, 2016). Quite possibly different results may be obtained by overexpressing one of the other 11 enzymatic activities of the Calvin cycle, and future efforts along these lines might benefit from starting with a modeling approach that predicts which of these activities is the most important one to overexpress.

\subsubsection{Trade-offs and limitations in metabolic engineering}

In order to engineer algae to produce more TAG, it is important to consider the trade-off between growth and TAG production. High cell growth rates and high lipid yields have long been considered to be mutually exclusive (Hu et al., 2008, Sheehan et al., 1998), since the synthesis of storage lipids such as TAG in algae is only stimulated under stress conditions where cell division has generally ceased. In agreement with this idea, Li et al. found that previously generated algal mutants with superior TAG accumulation generally had slower growth rates (Li et al., 2010a, Li et al., 2010b), which not only compromised TAG yield, but also increased the risk of contamination (Richmond, 2013). Recent advances in engineering algae for high lipid yield have produced some encouraging results where engineering of lipid catabolism (Trentacoste et al., 2013) and inhibition of starch synthesis (Breuer et al., 2014, de Jaeger et al., 2014) led to increases in lipid content without compromising growth. In the latter case the increase in TAG yield of Scenedesmus obliquus was further validated in controlled photobioreactors: the maximum TAG yield increased from about $0.14 \mathrm{~g}$ TAG/mol photon in the wild type to $0.22 \mathrm{~g}$ TAG/mol photon in a starchless mutant, representing a $51 \%$ increase; by contrast, no difference in photosynthetic efficiency was found between the wild type and mutant (Breuer et al., 2014).

In addition to improving TAG and biomass yield in microalgae, several other important challenges need to be addressed before algae will become a viable option for industrial-scale biofuel production. As presented by a practical assessment on the potential and feasibility of algal biofuel technology, these challenges are diverse and involve biological, technological, engineering, environmental, and techno-economic issues (Lundquist et al., 2010). To make algal biofuel costs more competitive with those for petroleum-based fuels, the investment in $R \& D$ from public and private sectors will need to focus on improving existing practices in large scale algae cultivation and crop protection, with integration of innovation in engineering technologies to enhance biomass and TAG productivity (Lundquist et al., 2010). Life Cycle Assessment (LCA) studies suggest that a positive energy balance in terms of net energy ratio (NER) or reduced net greenhouse gas (GHG) can be achieved in raceway pond algal cultivation systems (Collet et al., 2015, Slade and Bauen, 2013). However, a significant proportion of biofuel demands can be met only through creation of new large-scale algae cultivation infrastructures that are efficient in biomass harvesting, salt and N-recycling, and by developing new biorefinery processes for increasing downstream efficiency (Greenwell et al., 2010, Hannon et al., 2010, Lundquist et al., 2010).

\section{Conclusion and perspectives}


Directed efforts towards understanding the regulation of lipid metabolism at the physiological and genetic levels have brought us closer to a more efficient production of lipid-based biofuels and high value compounds. These efforts have been mainly guided by biochemical pathway analysis, and more recently by omics data that make it easier to identify genes that play critical roles in lipid metabolism. The results of such work have enabled metabolic engineering of microalgae with an experimental focus on lipid biosynthetic genes, genes that inhibit lipid turnover and pathways that compete with lipogenesis. The outcome of these efforts has been hit or miss; some have been modestly successful, others not successful at all. The time has come for a much more intensive effort to connect experimentation with modeling, starting with some serious attempts to model algal TAG production. However, with few exceptions, model predictions have not been tested experimentally, reflecting lack of collaborations among modelers and biologists in this field. Future engineering efforts should focus more on validation of the targets predicted by models (such as those described in Section 3), followed by iterative rounds of modeling and testing that lead to more accurate descriptions of algal metabolism. While few efforts have been made so far to test the manipulation of multiple genes simultaneously, modelers and experimenters working in conjunction should be able to make such strategies efficient and productive. This work should be increasingly done in industrial-relevant strains, using new genetic manipulation tools (such as those described in Section 4) that are making it possible to modify different microalgal species at will. In the future, biologists and modelers should also invest more effort into studying algal production under field conditions, where light and temperature fluctuate significantly more than they do in controlled laboratory conditions, and along with seasonal changes in diurnal rhythms can greatly impact algal photosynthesis and lipid synthesis.

Continued advances in high throughput multi-omics tools, computational modeling, and molecular genetic techniques should allow precise prediction and targeted modification of the TAG metabolic network (as depicted in Figure 1). This will enable a better understanding of photosynthetic carbon partitioning mechanisms between lipids, carbohydrates, and proteins, and how interconversion of membrane lipids and TAG are regulated. These integrated approaches will further facilitate elucidation of complex flux control as well as identification of rate limiting structural and regulatory genes. As a result, "designer" industrial algal strains with elevated TAG content and growth rates are within reach. Together with advances made by those who focus on culture techniques, harvesting, downstream processing, and LCA, the synergistic work of TAG modelers and experimenters should accelerate the commercialization of algal biofuels in ways that help address the energy and climate issues that are among the grandest challenges of science and engineering.

\section{Acknowledgments.}

This work was supported by an award from the National Science Foundation to S.M.M. (NSFEFRI-1332344) and awards to Y.L. from the National Science Foundation (CBET-1511939), Climate Change and Emissions Management Corporation (CCEMC), and Office of Naval Research (N00014-15-1-2219).

\section{References}


Babinger P,Kobl I,Mages W,Schmitt R. A link between DNA methylation and epigenetic silencing in transgenic Volvox carteri. Nucleic Acids Res. 2001;29:1261-71. Baroukh C,Munoz-Tamayo R,Bernard O,Steyer JP. Mathematical modeling of unicellular microalgae and cyanobacteria metabolism for biofuel production. Current Opinion in Biotechnology. 2015a;33:198-205. Baroukh C,Munoz-Tamayo R,Stever JP,Bernard O. A state of the art of metabolic networks of unicellular microalgae and cyanobacteria for biofuel production. Metabolic Engineering. 2015b;30:49-60.

Baroukh C,Muñoz-Tamayo R,Steyer J-P,Bernard O. DRUM: A New Framework for Metabolic Modeling under Non-Balanced Growth. Application to the Carbon Metabolism of Unicellular Microalgae. PLoS One. 2014;9:e104499. DYNAMIC FLUX BALANCE ANALYSIS FOR PREDICTING GENE OVEREXPRESSION EFFECTS IN BATCH CULTURES. Journal of Biological Systems. 2014;22:327-38.

Bellou S,Baeshen MN,Elazzazy AM,Aggeli D,Sayegh F,Aggelis G. Microalgal lipids biochemistry and biotechnological perspectives. Biotech Adv. 2014;32:1476-93. Benemann J. Microalgae for Biofuels and Animal Feeds. Energies. 2013;6:5869-86.

Blatti JL,Beld J,Behnke CA,Mendez M,Mayfield SP,Burkart MD. Manipulating fatty acid biosynthesis in microalgae for biofuel through protein-protein interactions. PLoS One. 2012;7:e42949.

Blatti JL,Michaud J,Burkart MD. Engineering fatty acid biosynthesis in microalgae for sustainable biodiesel. Curr Opin Chem Biol. 2013;17:496-505.

Boettcher M,McManus MT. Choosing the Right Tool for the Job: RNAi, TALEN, or CRISPR. Mol Cell. 2015;58:575-85.

Bolling C,Fiehn O. Metabolite profiling of Chlamydomonas reinhardtii under nutrient deprivation. Plant Physiol. 2005;139:1995-2005.

Boyle NR,Morgan JA. Flux balance analysis of primary metabolism in Chlamydomonas reinhardtii. BMC systems biology. 2009;3:4.

Boyle NR,Page MD,Liu B,Blaby IK,Casero D,Kropat J,et al. Three acyltransferases and nitrogenresponsive regulator are implicated in nitrogen starvation-induced triacylglycerol accumulation in Chlamydomonas. J Biol Chem. 2012;287:15811-25.

Boynton JE,Gillham NW,Harris EH,Hosler JP,Johnson AM,Jones AR,et al. Chloroplast Transformation in Chlamydomonas with High Velocity Microprojectiles. Science. 1988;240:1534-8.

Breuer G,de Jaeger L,Artus VPG,Martens DE,Springer J,Draaisma RB,et al. Superior triacylglycerol (TAG) accumulation in starchless mutants of Scenedesmus obliquus: (II) evaluation of TAG yield and productivity in controlled photobioreactors. Biotechnol Biofuels. 2014;7:11.

Carroll D. Genome engineering with zinc-finger nucleases. Genetics. 2011;188:773-82.

Cerutti H,Johnson AM,Gillham NW,Boynton JE. Epigenetic silencing of a foreign gene in nuclear transformants of Chlamydomonas. Plant Cell. 1997;9:925-45.

Chang HX,Huang Y,Fu Q,Liao Q,Zhu X. Kinetic characteristics and modeling of microalgae Chlorella vulgaris growth and $\mathrm{CO} 2$ biofixation considering the coupled effects of light intensity and dissolved inorganic carbon. Bioresour Technol. 2016;206:231-8.

Chang RL,Ghamsari L,Manichaikul A,Hom EFY,Balaji S,Fu W,et al. Metabolic network reconstruction of Chlamydomonas offers insight into light-driven algal metabolism. Molecular Systems Biology. 2011;7:1-13.

Chapman KD,Ohlrogge JB. Compartmentation of triacylglycerol accumulation in plants. J Biol Chem. 2012;287:2288-94. between astaxanthin and fatty acid biosynthesis in Haematococcus pluvialis (Chlorophyceae). The Plant Journal. 2015;81:95-107.

Chisti Y. Biodiesel from microalgae. Biotechnol Adv. 2007;25:294-306.

Choi YE,Hwang H,Kim HS,Ahn JW,Jeong WJ,Yang JW. Comparative proteomics using lipid overproducing or less-producing mutants unravels lipid metabolisms in Chlamydomonas reinhardtii. Bioresour Technol. 2013;145:108-15. 
Cogne G,Rügen M,Bockmayr A,Titica M,Dussap CG,Cornet JF,et al. A model-based method for investigating bioenergetic processes in autotrophically growing eukaryotic microalgae: Application to the green algae Chlamydomonas reinhardtii. Biotechnology progress. 2011;27:631-40.

Cohen Z,Khozin-Goldberg I,Adlerstein D,Bigogno C. The role of triacylglycerol as a reservoir of polyunsaturated fatty acids for the rapid production of chloroplastic lipids in certain microalgae. Biochemical Society Transactions. 2000;28:740-3.

Collet P,Hélias A,Lardon L,Steyer J-P,Bernard O. Recommendations for Life Cycle Assessment of algal fuels. Applied Energy. 2015;154:1089-102.

Cronan JE, Jr.,Waldrop GL. Multi-subunit acetyl-CoA carboxylases. Prog Lipid Res. 2002;41:407-35.

Daboussi F,Leduc S,Marechal A,Dubois G,Guyot V,Perez-Michaut C,et al. Genome engineering empowers the diatom Phaeodactylum tricornutum for biotechnology. Nat Commun. 2014;5:3831.

Dal'Molin CGD,Quek LE,Palfreyman RW,Nielsen LK. AlgaGEM - a genome-scale metabolic reconstruction of algae based on the Chlamydomonas reinhardtii genome. Bmc Genomics. 2011;12:10.

De Bhowmick G,Koduru L,Sen R. Metabolic pathway engineering towards enhancing microalgal lipid biosynthesis for biofuel application-A review. Renewable \& Sustainable Energy Reviews. 2015;50:123953.

de Jaeger L,Verbeek R,Springer J,Eggink G,Wijffels RH. Superior triacylglycerol (TAG) accumulation in starchless mutants of Scenedesmus obliquus: (I) mutant generation and characterisation. Biotechnology for Biofuels. 2014;7.

de Mooij T,Janssen M,Cerezo-Chinarro O,Mussgnug J,Kruse O,Ballottari M,et al. Antenna size reduction as a strategy to increase biomass productivity: a great potential not yet realized. Journal of Applied Phycology. 2015;27:1063-77.

Dejtisakdi W,Miller SM. Overexpression of Calvin cycle enzyme fructose 1,6-bisphosphatase in Chlamydomonas reinhardtii has a detrimental effect on growth. Algal Research. 2016;14:116-26.

Deng X,Cai J,Fei X. Effect of the expression and knockdown of citrate synthase gene on carbon flux during triacylglycerol biosynthesis by green algae Chlamydomonas reinhardtii. BMC Biochem. 2013;14:38.

Deng X,Cai J,Li Y,Fei X. Expression and knockdown of the PEPC1 gene affect carbon flux in the biosynthesis of triacylglycerols by the green alga Chlamydomonas reinhardtii. Biotechnol Lett. 2014;36:2199-208.

Deng XD,Gu B,Li YJ,Hu XW,Guo JC,Fei XW. The roles of acyl-CoA: diacylglycerol acyltransferase 2 genes in the biosynthesis of triacylglycerols by the green algae Chlamydomonas reinhardtii. Mol Plant. 2012;5:945-7.

DOE U. National algal biofuels technology roadmap. US Department of Energy, Office of Energy Efficiency and Renewable Energy, Biomass Program. 2010.

Donnelly ML,Luke G,Mehrotra A,Li X,Hughes LE,Gani D,et al. Analysis of the aphthovirus 2A/2B polyprotein 'cleavage' mechanism indicates not a proteolytic reaction, but a novel translational effect: a putative ribosomal 'skip'. J Gen Virol. 2001;82:1013-25.

Doudna JA,Charpentier E. Genome editing. The new frontier of genome engineering with CRISPR-Cas9. Science. 2014;346:1258096.

Dunahay T,Jarvis E,Dais S,Roessler P. Manipulation of microalgal lipid production using genetic engineering. Applied Biochemistry and Biotechnology. 1996;57-58:223-31.

Dunahay TG,Jarvis EE,Roessler PG. Genetic transformation of the diatoms Cyclotella cryptica and Navicula saprophila. J Phycol. 1995;31:1004-12.

Edwards JS,Covert M,Palsson B. Metabolic modelling of microbes: the flux-balance approach. Environ Microbiol. 2002;4:133-40.

Engel TR, P. Thermodynamics, Statistical Thermodynamics, and Kinetics. San Francisco: Pearson Education - Benjamin Cummings; 2006.

Fan J,Ning K,Zeng X,Luo Y,Wang D,Hu J,et al. Genomic Foundation of Starch-to-Lipid Switch in Oleaginous Chlorella spp. Plant Physiol. 2015;169:2444-61. 
Fan JL,Andre C,Xu CC. A chloroplast pathway for the de novo biosynthesis of triacylglycerol in Chlamydomonas reinhardtii. FEBS Lett. 2011;585:1985-91.

Feng L,Wang K,Li Y,Tan Y,Kong J,Li H,et al. Overexpression of SBPase enhances photosynthesis against high temperature stress in transgenic rice plants. Plant Cell Rep. 2007;26:1635-46. Gaj T,Gersbach CA,Barbas CF, 3rd. ZFN, TALEN, and CRISPR/Cas-based methods for genome engineering. Trends Biotechnol. 2013;31:397-405.

Gao H, Wright, D.A., Li, T., Wang, Y., Horken, K., Weeks, D.P., Yang, B., Spalding, M.H. TALE activation of endogenous genes in Chlamydomonas reinhardtii. Algal Research. 2014;5:52-60.

Georgianna DR,Mayfield SP. Exploiting diversity and synthetic biology for the production of algal biofuels. Nature. 2012;488:329-35.

Gilbert TRK, R. V.; Foster, N.; Davies, G. Chemistry: The science in context. New York: W. W. Norton \& Company; 2009.

Goodson C,Roth R,Wang ZT,Goodenough U. Structural Correlates of Cytoplasmic and Chloroplast Lipid Body Synthesis in Chlamydomonas reinhardtii and Stimulation of Lipid Body Production with Acetate Boost. Eukaryot Cell. 2011;10:1592-606.

Grafahrend-Belau E,Junker A,Eschenröder A,Müller J,Schreiber F,Junker BH. Multiscale metabolic modeling: dynamic flux balance analysis on a whole-plant scale. Plant Physiol. 2013;163:637-47.

Greenwell HC,Laurens LM,Shields RJ,Lovitt RW,Flynn KJ. Placing microalgae on the biofuels priority list: a review of the technological challenges. J R Soc Interface. 2010;7:703-26.

Guarnieri MT,Nag A,Smolinski SL,Darzins A,Seibert M,Pienkos PT. Examination of triacylglycerol biosynthetic pathways via de novo transcriptomic and proteomic analyses in an unsequenced microalga. PLoS One. 2011;6:e25851.

Guiheneuf F,Leu S,Zarka A,Khozin-Goldberg I,Khalilov I,Boussiba S. Cloning and molecular characterization of a novel acyl-CoA:diacylglycerol acyltransferase 1-like gene (PtDGAT1) from the diatom Phaeodactylum tricornutum. The FEBS journal. 2011;278:3651-66.

Hagen C,Braune W, Greulich F. Functional aspects of secondary carotenoids in Haematococcus lacustris [Girod] Rostafinski (Volvocales) IV. Protection from photodynamic damage. Journal of Photochemistry and Photobiology B: Biology. 1993;20:153-60.

Hannon M,Gimpel J,Tran M,Rasala B,Mayfield S. Biofuels from algae: challenges and potential. Biofuels. 2010;1:763-84.

Harvey M,Pilgrim S. The new competition for land: Food, energy, and climate change. Food Policy. 2011;36, Supplement 1:S40-S51.

Hilton IB,Gersbach CA. Enabling functional genomics with genome engineering. Genome Res. 2015;25:1442-55.

Hoffmann M,Wagner M,Abbadi A,Fulda M,Feussner I. Metabolic engineering of omega3-very long chain polyunsaturated fatty acid production by an exclusively acyl-CoA-dependent pathway. The Journal of biological chemistry. 2008;283:22352-62.

Hohenschuh W,Hector R,Murthy GS. A dynamic flux balance model and bottleneck identification of glucose, xylose, xylulose co-fermentation in Saccharomyces cerevisiae. Bioresour Technol. 2015;188:153-60.

Holthuis JC,Menon AK. Lipid landscapes and pipelines in membrane homeostasis. Nature. 2014;510:4857.

Hsieh HJ,Su CH,Chien LJ. Accumulation of lipid production in Chlorella minutissima by triacylglycerol biosynthesis-related genes cloned from Saccharomyces cerevisiae and Yarrowia lipolytica. Journal of microbiology. 2012;50:526-34.

$\mathrm{Hu}$ Q,Sommerfeld M,Jarvis E,Ghirardi M,Posewitz M,Seibert M,et al. Microalgal triacylglycerols as feedstocks for biofuel production: perspectives and advances. Plant J. 2008;54:621-39.

Huerlimann R,Heimann K. Comprehensive guide to acetyl-carboxylases in algae. Crit Rev Biotechnol. 2013;33:49-65. 
Hwangbo K,Ahn J-W,Lim J-M,Park Y-I,Liu J,Jeong W-J. Overexpression of stearoyl-ACP desaturase enhances accumulations of oleic acid in the green alga Chlamydomonas reinhardtii. Plant Biotechnology Reports. 2014;8:135-42.

Ibanez-Salazar A,Rosales-Mendoza S,Rocha-Uribe A,Ramirez-Alonso JI,Lara-Hernandez I,HernandezTorres A,et al. Over-expression of Dof-type transcription factor increases lipid production in Chlamydomonas reinhardtii. J Biotechnol. 2014;184:27-38.

Ichikawa Y,Tamoi M,Sakuyama H,Maruta T,Ashida H,Yokota A,et al. Generation of transplastomic lettuce with enhanced growth and high yield. GM Crops. 2010;1:322-6.

Imam S,Schauble S,Valenzuela J,de Lomana ALG,Carter W,Price ND,et al. A refined genome-scale reconstruction of Chlamydomonas metabolism provides a platform for systems-level analyses. Plant $\mathrm{J}$. 2015;84:1239-56.

Iskandarov U,Sitnik S,Shtaida N,Didi-Cohen S,Leu S,Khozin-Goldberg I,et al. Cloning and characterization of a GPAT-like gene from the microalga Lobosphaera incisa (Trebouxiophyceae): overexpression in Chlamydomonas reinhardtii enhances TAG production. Journal of Applied Phycology. 2015;28:907-19.

Iwai M,Ikeda K,Shimojima M,Ohta H. Enhancement of extraplastidic oil synthesis in Chlamydomonas reinhardtii using a type-2 diacylglycerol acyltransferase with a phosphorus starvation-inducible promoter. Plant Biotechnol J. 2014;12:808-19.

James ES,Cronan JE. Expression of two Escherichia coli acetyl-CoA carboxylase subunits is autoregulated. Journal of Biological Chemistry. 2004;279:2520-7.

Jia J,Han D,Gerken HG,Li Y,Sommerfeld M,Hu Q,et al. Molecular mechanisms for photosynthetic carbon partitioning into storage neutral lipids in Nannochloropsis oceanica under nitrogen-depletion conditions. Algal Research. 2015;7:66-77.

Jiang W,Brueggeman AJ,Horken KM,Plucinak TM,Weeks DP. Successful transient expression of Cas9 and single guide RNA genes in Chlamydomonas reinhardtii. Eukaryot Cell. 2014;13:1465-9.

Jinek M,Chylinski K,Fonfara I,Hauer M,Doudna JA,Charpentier E. A programmable dual-RNA-guided DNA endonuclease in adaptive bacterial immunity. Science. 2012;337:816-21.

Jinkerson RE,Jonikas MC. Molecular techniques to interrogate and edit the Chlamydomonas nuclear genome. Plant J. 2015;82:393-412.

Johnson X,Alric J. Central carbon metabolism and electron transport in Chlamydomonas reinhardtii: metabolic constraints for carbon partitioning between oil and starch. Eukaryot Cell. 2013;12:776-93.

Kalscheuer R,Steinbuchel A. A novel bifunctional wax ester synthase/acyl-CoA:diacylglycerol acyltransferase mediates wax ester and triacylglycerol biosynthesis in Acinetobacter calcoaceticus ADP1. The Journal of biological chemistry. 2003;278:8075-82.

Kauffman KJ,Prakash P,Edwards JS. Advances in flux balance analysis. Current Opinion in Biotechnology. 2003; 14:491-6.

Kaye Y GO, Leu S, Zarka A, Zorin B , Didi-Cohen S, Khozin-Goldberg I and Boussiba S. Metabolic engineering toward enhanced LC-PUFA biosynthesis in Nannochloropsis oceanica: Overexpression of endogenous $\Delta 12$ desaturase driven by stress-inducible promoter leads to enhanced deposition of polyunsaturated fatty acids in TAG. Algal Research. 2015.

Klaus D,Ohlrogge JB,Neuhaus HE,Dormann P. Increased fatty acid production in potato by engineering of acetyl-CoA carboxylase. Planta. 2004;219:389-96.

Kleessen S,Irgang S,Klie S,Giavalisco P,Nikoloski Z. Integration of transcriptomics and metabolomics data specifies the metabolic response of Chlamydomonas to rapamycin treatment. The Plant Journal. 2015;81:822-35.

Kliphuis AM,Klok AJ,Martens DE,Lamers PP,Janssen M,Wijffels RH. Metabolic modeling of Chlamydomonas reinhardtii: energy requirements for photoautotrophic growth and maintenance. Journal of applied phycology. 2012;24:253-66.

Knies D,Wittmuss P,Appel S,Sawodny O,Ederer M,Feuer R. Modeling and Simulation of Optimal Resource Management during the Diurnal Cycle in Emiliania huxleyi by Genome-Scale Reconstruction and an Extended Flux Balance Analysis Approach. Metabolites. 2015;5:659-76. 
Kohlwein SD. Triacylglycerol homeostasis: insights from yeast. J Biol Chem. 2010;285:15663-7. Kong F,Yamasaki T,Kurniasih SD,Hou L,Li X,Ivanova N,et al. Robust expression of heterologous genes by selection marker fusion system in improved Chlamydomonas strains. J Biosci Bioeng. 2015;120:23945.

Kozaki A,Mayumi K,Sasaki Y. Thiol-disulfide exchange between nuclear-encoded and chloroplastencoded subunits of pea acetyl-CoA carboxylase. The Journal of biological chemistry. 2001;276:3991925.

La Russa M,Bogen C,Uhmeyer A,Doebbe A,Filippone E,Kruse O,et al. Functional analysis of three type-

2 DGAT homologue genes for triacylglycerol production in the green microalga Chlamydomonas reinhardtii. J Biotechnol. 2012;162:13-20.

Leyva L,Bashan Y,de-Bashan L. Activity of acetyl-CoA carboxylase is not directly linked to accumulation of lipids when Chlorella vulgaris is co-immobilised with Azospirillum brasilense in alginate under autotrophic and heterotrophic conditions. Annals of Microbiology. 2015;65:339-49.

Li-Beisson Y,Beisson F, Riekhof W. Metabolism of acyl-lipids in Chlamydomonas reinhardtii. The Plant Journal. 2015;82:504-22.

Li-Beisson Y,Shorrosh B,Beisson F,Andersson MX,Arondel V,Bates PD,et al. Acyl-lipid metabolism. Arabidopsis Book. 2013;11:e0161. Nannochloropsis Reveals the Mechanisms of Oil Synthesis in Microalgae. Plant Cell. 2014;26:1645-65. Li X,Moellering ER,Liu B,Johnny C,Fedewa M,Sears BB,et al. A galactoglycerolipid lipase is required for triacylglycerol accumulation and survival following nitrogen deprivation in Chlamydomonas reinhardtii. The Plant cell. 2012;24:4670-86.

Li Y,Han D,Hu G,Dauvillee D,Sommerfeld M,Ball S, et al. Chlamydomonas starchless mutant defective in ADP-glucose pyrophosphorylase hyper-accumulates triacylglycerol. Metab Eng. 2010a;12:387-91.

Li Y,Han D,Hu G,Sommerfeld M,Hu Q. Inhibition of starch synthesis results in overproduction of lipids in Chlamydomonas reinhardtii. Biotechnol Bioeng. 2010b;107:258-68.

Li Y,Han D,Yoon K,Zhu S,Sommerfeld M,Hu Q. Molecular and Cellular Mechanisms for Lipid Synthesis and Accumulation in Microalgae: Biotechnological Implications. Handbook of Microalgal Culture: John Wiley \& Sons, Ltd; 2013. p. 545-65.

Li Y,Sommerfeld M,Chen F,Hu Q. Consumption of oxygen by astaxanthin biosynthesis: a protective mechanism against oxidative stress in Haematococcus pluvialis (Chlorophyceae). J Plant Physiol. 2008;165:1783-97.

Liu B,Benning C. Lipid metabolism in microalgae distinguishes itself. Curr Opin Biotechnol. 2012;24:300-9.

Liu J,Han D,Yoon K,Hu Q,Li Y. Characterization of type 2 diacylglycerol acyltransferases in Chlamydomonas reinhardtii reveals their distinct substrate specificities and functions in triacylglycerol biosynthesis. The Plant Journal. 2016;86:3-19.

Luke G,Escuin H,De Felipe P,Ryan M. 2A to the fore - research, technology and applications. Biotechnol Genet Eng Rev. 2010;26:223-60.

Lundquist TJ, Woertz IC, Quinn N,Benemann JR. A realistic technology and engineering assessment of algae biofuel production. Energy Biosciences Institute. 2010:1.

Lung SC,Weselake RJ. Diacylglycerol acyltransferase: A key mediator of plant triacylglycerol synthesis. Lipids. 2006;41:1073-88.

Lv H,Qu G,Qi X,Lu L,Tian C,Ma Y. Transcriptome analysis of Chlamydomonas reinhardtii during the process of lipid accumulation. Genomics. 2013;101:229-37.

1401 Lykidis A,Ivanova N. Genomic prospecting for microbial biodiesel production. Washington: ASM Press.

Mahadevan R,Edwards JS,Doyle FJ. Dynamic flux balance analysis of diauxic growth in Escherichia coli. Biophysical journal. 2002;83:1331-40. 
Manandhar-Shrestha K,Hildebrand M. Characterization and manipulation of a DGAT2 from the diatom Thalassiosira pseudonana: Improved TAG accumulation without detriment to growth, and implications for chloroplast TAG accumulation. Algal Research. 2015;12:239-48.

Manichaikul A,Ghamsari L,Hom EF,Lin C,Murray RR,Chang RL,et al. Metabolic network analysis integrated with transcript verification for sequenced genomes. Nature methods. 2009;6:589-92.

May P,Christian JO,Kempa S, Walther D. ChlamyCyc: an integrative systems biology database and webportal for Chlamydomonas reinhardtii. BMC Genomics. 2009;10:209.

May P,Wienkoop S,Kempa S,Usadel B,Christian N,Rupprecht J,et al. Metabolomics- and proteomicsassisted genome annotation and analysis of the draft metabolic network of Chlamydomonas reinhardtii. Genetics. 2008;179:157-66.

Merchant SS,Kropat J,Liu B,Shaw J,Warakanont J. TAG, you're it! Chlamydomonas as a reference organism for understanding algal triacylglycerol accumulation. Curr Opin Biotechnol. 2012;23:352-63.

Merchant SS,Prochnik SE,Vallon O,Harris EH,Karpowicz SJ,Witman GB,et al. The Chlamydomonas genome reveals the evolution of key animal and plant functions. Science. 2007;318:245-50.

Miller R,Wu G,Deshpande RR,Vieler A,Gartner K,Li X,et al. Changes in transcript abundance in Chlamydomonas reinhardtii following nitrogen deprivation predict diversion of metabolism. Plant Physiol. 2010;154:1737-52.

Miyagawa Y,Tamoi M,Shigeoka S. Overexpression of a cyanobacterial fructose-1,6-/sedoheptulose-1,7bisphosphatase in tobacco enhances photosynthesis and growth. Nat Biotechnol. 2001;19:965-9.

Moellering ER,Benning C. RNA interference silencing of a major lipid droplet protein affects lipid droplet size in Chlamydomonas reinhardtii. Eukaryot Cell. 2009;9:97-106.

Mühlroth A,Li K,Røkke G,Winge P,Olsen Y,Hohmann-Marriott MF,et al. Pathways of lipid metabolism in marine algae, co-expression network, bottlenecks and candidate genes for enhanced production of EPA and DHA in species of Chromista. Mar Drugs. 2013;11:4662-97.

Naldini L. Ex vivo gene transfer and correction for cell-based therapies. Nat Rev Genet. 2011;12:301-15. Nguyen HM,Baudet M,Cuine S,Adriano JM,Barthe D,Billon E,et al. Proteomic profiling of oil bodies isolated from the unicellular green microalga Chlamydomonas reinhardtii: with focus on proteins involved in lipid metabolism. Proteomics. 2011;11:4266-73.

Nikoloski Z,Perez-Storey R,Sweetlove LJ. Inference and prediction of metabolic network fluxes. Plant Physiol. 2015;169:1443-55.

Niu YF,Zhang MH,Li DW,Yang WD,Liu JS,Bai WB,et al. Improvement of Neutral Lipid and Polyunsaturated Fatty Acid Biosynthesis by Overexpressing a Type 2 Diacylglycerol Acyltransferase in Marine Diatom Phaeodactylum tricornutum. Marine Drugs. 2013;11:4558-69.

Ohlrogge J,Browse J. Lipid biosynthesis. Plant Cell. 1995;7:957-70.

Ohlrogge JB,Jaworski JG. Regulation of Fatty Acid Synthesis. Annual review of plant physiology and plant molecular biology. 1997;48:109-36.

Ort DR,Melis A. Optimizing antenna size to maximize photosynthetic efficiency. Plant Physiol. 2011;155:79-85.

Orth JD, Thiele I,Palsson BO. What is flux balance analysis? Nature Biotechnology. 2010;28:245-8.

Packer A,Li Y,Andersen T,Hu Q,Kuang Y,Sommerfeld M. Growth and neutral lipid synthesis in green microalgae: a mathematical model. Bioresour Technol. 2010;102:111-7.

Plucinak TM,Horken KM,Jiang W,Fostvedt J,Nguyen ST,Weeks DP. Improved and versatile viral 2A platforms for dependable and inducible high-level expression of dicistronic nuclear genes in Chlamydomonas reinhardtii. Plant J. 2015;82:717-29.

Polle JE,Kanakagiri SD,Melis A. tla1, a DNA insertional transformant of the green alga Chlamydomonas reinhardtii with a truncated light-harvesting chlorophyll antenna size. Planta. 2003;217:49-59.

Radakovits R,Eduafo PM,Posewitz MC. Genetic engineering of fatty acid chain length in Phaeodactylum tricornutum. Metabolic engineering. 2011;13:89-95.

Radakovits R,Jinkerson RE,Darzins A,Posewitz MC. Genetic engineering of algae for enhanced biofuel production. Eukaryot Cell. 2010;9:486-501. 
Radakovits R,Jinkerson RE,Fuerstenberg SI,Tae H,Settlage RE,Boore JL,et al. Draft genome sequence and genetic transformation of the oleaginous alga Nannochloropis gaditana. Nat Commun. 2012;3:686. Raman K,Chandra N. Flux balance analysis of biological systems: applications and challenges. Briefings in bioinformatics. 2009;10:435-49.

Rasala BA,Lee PA,Shen Z,Briggs SP,Mendez M,Mayfield SP. Robust expression and secretion of Xylanase1 in Chlamydomonas reinhardtii by fusion to a selection gene and processing with the FMDV 2A peptide. PLoS One. 2012;7:e43349.

Rasala BA,Muto M,Lee PA,Jager M,Cardoso RMF,Behnke CA,et al. Production of therapeutic proteins in algae, analysis of expression of seven human proteins in the chloroplast of Chlamydomonas reinhardtii. Plant biotechnology journal. 2010;8:719-33.

Recht L,Topfer N,Batushansky A,Sikron N,Gibon Y,Fait A,et al. Metabolite profiling and integrative modeling reveal metabolic constraints for carbon partitioning under nitrogen starvation in the green algae Haematococcus pluvialis. J Biol Chem. 2014;289:30387-403.

Reijnders MJ,van Heck RG,Lam CM,Scaife MA,dos Santos VA,Smith AG,et al. Green genes: bioinformatics and systems-biology innovations drive algal biotechnology. Trends Biotechnol. 2014;32:617-26.

Rezanka T,Nedbalova L,Prochazkova L,Sigler K. Lipidomic profiling of snow algae by ESI-MS and silver-LC/APCI-MS. Phytochemistry. 2014;100:34-42.

Richmond A. Biological Principles of Mass Cultivation of Photoautotrophic Microalgae. Handbook of Microalgal Culture: John Wiley \& Sons, Ltd; 2013. p. 169-204.

Saha S,Enugutti B,Rajakumari S,Rajasekharan R. Cytosolic triacylglycerol biosynthetic pathway in oilseeds. Molecular cloning and expression of peanut cytosolic diacylglycerol acyltransferase. Plant Physiol. 2006;141:1533-43.

Sanjaya,Miller R,Durrett TP,Kosma DK,Lydic TA,Muthan B,et al. Altered lipid composition and enhanced nutritional value of Arabidopsis leaves following introduction of an algal diacylglycerol acyltransferase 2. The Plant cell. 2013;25:677-93.

Scaife MA,Nguyen GT,Rico J,Lambert D,Helliwell KE,Smith AG. Establishing Chlamydomonas reinhardtii as an industrial biotechnology host. The Plant Journal. 2015:532-46.

Sheehan J,Dunahay T,Benemann J,Roessler PG. US Department of Energy's Office of Fuels Development, July 1998. A Look Back at the US Department of Energy's Aquatic Species Program Biodiesel from Algae, Close Out Report TP-580-24190. Golden, CO: National Renewable Energy Laboratory. 1998.

Simionato D,Block MA,La Rocca N,Jouhet J,Marechal E,Finazzi G,et al. The response of Nannochloropsis gaditana to nitrogen starvation includes de novo biosynthesis of triacylglycerols, a decrease of chloroplast galactolipids, and reorganization of the photosynthetic apparatus. Eukaryot Cell. 2013;12:665-76.

Sizova I,Greiner A,Awasthi M,Kateriya S,Hegemann P. Nuclear gene targeting in Chlamydomonas using engineered zinc-finger nucleases. Plant J. 2013;73:873-82.

Slade R,Bauen A. Micro-algae cultivation for biofuels: Cost, energy balance, environmental impacts and future prospects. Biomass and Bioenergy. 2013;53:29-38.

Tamoi M,Nagaoka M,Miyagawa Y,Shigeoka S. Contribution of fructose-1,6-bisphosphatase and sedoheptulose-1,7-bisphosphatase to the photosynthetic rate and carbon flow in the Calvin cycle in transgenic plants. Plant Cell Physiol. 2006;47:380-90.

Terashima M,Specht M,Hippler M. The chloroplast proteome: a survey from the Chlamydomonas reinhardtii perspective with a focus on distinctive features. Curr Genet. 2011;57:151-68.

Tevatia R,Demirel Y,Blum P. Kinetic modeling of photoautotropic growth and neutral lipid accumulation in terms of ammonium concentration in Chlamydomonas reinhardtii. Bioresour Technol. 2012;119:41924.

Tonon T,Harvey D,Larson TR,Graham IA. Long chain polyunsaturated fatty acid production and partitioning to triacylglycerols in four microalgae. Phytochemistry. 2002;61:15-24. 
1505

1506

1507

1508

1509

1510

1511

1512

1513

1514

1515

1516

1517

1518

1519

1520

1521

1522

1523

1524

1525

1526

1527

1528

1529

1530

1531

1532

1533

1534

1535

1536

1537

1538

1539

1540

1541

1542

1543

1544

1545

1546

1547

1548

1549

1550

1551

1552

1553

1554

Trentacoste EM,Shrestha RP,Smith SR,Gle C,Hartmann AC,Hildebrand M,et al. Metabolic engineering of lipid catabolism increases microalgal lipid accumulation without compromising growth. Proc Natl Acad Sci U S A. 2013;110:19748-53.

Tsai C-H,Zienkiewicz K,Amstutz CL,Brink BG,Warakanont J,Roston R,et al. Dynamics of protein and polar lipid recruitment during lipid droplet assembly in Chlamydomonas reinhardtii. The Plant Journal. 2015;83:650-60.

Tsai CH,Warakanont J,Takeuchi T,Sears BB,Moellering ER,Benning C. The protein Compromised Hydrolysis of Triacylglycerols 7 (CHT7) acts as a repressor of cellular quiescence in Chlamydomonas. Proc Natl Acad Sci U S A. 2014;111:15833-8.

Ullah M,Schmidt H,Cho K-H,Wolkenhauer O. Deterministic modelling and stochastic simulation of biochemical pathways using MATLAB. IEE Proceedings-Systems Biology. 2006;153:53-60.

Vieler A,Wu G,Tsai CH,Bullard B,Cornish AJ,Harvey C,et al. Genome, functional gene annotation, and nuclear transformation of the heterokont oleaginous alga Nannochloropsis oceanica CCMP1779. PLoS genetics. 2012;8:e1003064.

Wagner M,Hoppe K,Czabany T,Heilmann M,Daum G,Feussner I,et al. Identification and characterization of an acyl-CoA:diacylglycerol acyltransferase 2 (DGAT2) gene from the microalga O. tauri. Plant physiology and biochemistry : PPB / Societe francaise de physiologie vegetale. 2010;48:407-16.

Wan M,Liu P,Xia J,Rosenberg JN,Oyler GA,Betenbaugh MJ,et al. The effect of mixotrophy on microalgal growth, lipid content, and expression levels of three pathway genes in Chlorella sorokiniana. Applied microbiology and biotechnology. 2011;91:835-44.

Wang D,Ning K,Li J,Hu J,Han D,Wang H,et al. Nannochloropsis genomes reveal evolution of microalgal oleaginous traits. PLoS Genet. 2014;10:e1004094.

Wang HX,Alvarez S,Hicks LM. Comprehensive Comparison of iTRAQ and Label-free LC-Based Quantitative Proteomics Approaches Using Two Chlamydomonas reinhardtii Strains of Interest for Biofuels Engineering. Journal of Proteome Research. 2012;11:487-501.

Wang X,Liu Y-H,Hu D-X,Balamurugan S,Lu Y,Yang W-D,et al. Identification of a putative patatin-like phospholipase domain-containing protein 3 (PNPLA3) ortholog involved in lipid metabolism in microalga Phaeodactylum tricornutum. Algal Research. 2015;12:274-9.

Wase N,Tu B,Black PN,DiRusso CC. Phenotypic screening identifies Brefeldin A/Ascotoxin as an inducer of lipid storage in the algae Chlamydomonas reinhardtii. Algal Research. 2015;11:74-84.

Weyman PD,Beeri K,Lefebvre SC,Rivera J,McCarthy JK,Heuberger AL,et al. Inactivation of Phaeodactylum tricornutum urease gene using transcription activator-like effector nuclease-based targeted mutagenesis. Plant Biotechnol J. 2015;13:460-70.

Wilhelm C,Buechel C,Fisahn J,Goss R,Jakob T,LaRoche J,et al. The regulation of carbon and nutrient assimilation in diatoms is significantly different from green algae. Protist. 2006;157:91-124.

Wu C,Xiong W,Dai J,Wu Q. Genome-Based Metabolic Mapping and 13C Flux Analysis Reveal Systematic Properties of an Oleaginous Microalga Chlorella protothecoides. Plant Physiol. 2015;167:58699.

Yang J,Rasa E,Tantayotai P,Scow KM,Yuan H,Hristova KR. Mathematical model of Chlorella minutissima UTEX2341 growth and lipid production under photoheterotrophic fermentation conditions. Bioresour Technol. 2010;102:3077-82.

Yoon K,Han D,Li Y,Sommerfeld M,Hu Q. Phospholipid:diacylglycerol acyltransferase is a multifunctional enzyme involved in membrane lipid turnover and degradation while synthesizing triacylglycerol in the unicellular green microalga Chlamydomonas reinhardtii. Plant Cell. 2012;24:370824.

Yuan L,Voelker TA,Hawkins DJ. Modification of the substrate specificity of an acyl-acyl carrier protein thioesterase by protein engineering. Proceedings of the National Academy of Sciences of the United States of America. 1995;92:10639-43.

Zalutskaya Z,Kharatyan N,Forchhammer K,Ermilova E. Reduction of PII signaling protein enhances lipid body production in Chlamydomonas reinhardtii. Plant Science. 2015;240:1-9. 
Zou JT,Wei YD,Jako C,Kumar A,Selvaraj G,Taylor DC. The Arabidopsis thaliana TAG1 mutant has a mutation in a diacylglycerol acyltransferase gene. Plant J. 1999;19:645-53.

\section{Figure Legends}

Figure 1 Rational algae engineering for production of biofuels and high-value products. Schematic diagram indicates how experimental work (informed by molecular, biochemical, and omics data; upper left) and various kinds of computational modeling approaches (lower left) can both contribute to our understanding of algal TAG biosynthesis, but when experimental work and modeling are integrated they can work synergistically toward this goal. Combined with powerful new molecular genetic tools (middle), these approaches should make rational, targeted genetic modifications of microalgae for enhanced TAG production a reality (right).

\section{Figure 2 FA and TAG biosynthesis pathways in microalgae.}

FA/TAG biosynthesis in microalgae involves coordinated crosstalk among multiple pathways in multiple subcellular compartments including the endoplasmic reticulum and plastid. For FA and TAG biosynthesis, energy (ATP) and reducing power (NADH and NADPH) provided by photosynthesis drive the synthesis of 3-phosphoglycerate (3-PGA) in the Calvin cycle. In some microalgae, 3-PGA can also be derived from the storage molecule starch via several reactions, including the one catalyzed by ADP-glucose pyrophosphorylase (AGPase). 3-PGA is converted to acetyl-CoA (through intermediates phosphoenolpyruvate and pyruvate, not shown), which is the precursor of de novo lipid synthesis in the chloroplast. FA/TAG biosynthesis begins with carboxylation of acetyl-CoA to form malonyl-CoA, catalyzed by the acetyl-CoA carboxylase (ACCase) enzyme complex. FA chains are elongated by the action of 3-ketoacyl ACP (acyl carrier protein), or KAS enzymes. The resulting acyl-ACP chains can enter the Kennedy pathway of TAG biosynthesis in the chloroplast. Alternatively, when acyl elongation reactions are terminated, the released free fatty acids are transported to the outer membrane of the chloroplast, where they can be reactivated and exported as acyl-CoA, serving as substrate in the ER-localized Kennedy pathway. Kennedy pathway intermediates and enzymes include lysophosphatidic acid (lyso PA), phosphatidic acid (PA), diacylglyerol (DAG), glycerol-3phosphate acyltransferase (GPAT), lysophosphatidic acid acyltransferase (LPAAT), phosphatidic acid phosphatase (PAP), and diacylglycerol acyltransferase (DGAT). Apart from the Kennedy pathway, an acyl-CoA-independent pathway of TAG biosynthesis catalyzed by PDATs (phospholipid:diacylglycerol acyltransferases) produces TAG by transferring a fatty acyl moiety from a phospholipid to DAG. Dashed arrows indicate multi-step reactions. Question marks indicate enzymes or reactions that are not established to exist in the chloroplast of microalgae. Membrane lipid abbreviations: PC, phosphatidylchloline; PE: phosphatidylethanolamine; PG: phosphatidylglycerol; PI: phosphatidylinositol; LD: lipid droplets, ENR, enoyl-ACP reductase; FAT, fatty acyl-ACP thioesterase; HD, 3-hydroxyacylACP dehydratase; KAR, 3-ketoacyl-ACP reductase (modified from (Li et al., 2013)). 
Table 1 Pros and cons of the different types of computational models of microalgae

\begin{tabular}{|l|l|l|}
\hline \multicolumn{1}{|c|}{ Model Type } & \multicolumn{1}{c|}{ Pros } & \multicolumn{1}{c|}{ Cons } \\
\hline FBA & $\begin{array}{l}\text { - Thousands of reactions } \\
\text { can be computed quickly, } \\
\text { allowing entire metabolic } \\
\text { networks to be described }\end{array}$ & $\begin{array}{l}\text { - Concentrations of } \\
\text { metabolites cannot be } \\
\text { determined } \\
\text { Biologically relevant } \\
\text { objective functions are } \\
\text { difficult to generate }\end{array}$ \\
\hline MFA & $\begin{array}{l}\text { - Experimental data are } \\
\text { used to constrain possible } \\
\text { solutions }\end{array}$ & $\begin{array}{l}\text { - Are limited to describing } \\
\text { metabolites for which } \\
\text { experimentally } \\
\text { determined information is } \\
\text { available }\end{array}$ \\
\hline DRUM & $\begin{array}{l}\text { - Useful for microalgae } \\
\text { with genomes that have } \\
\text { not been sequenced } \\
\text { Can describe diurnal } \\
\text { cycle of microalgae } \\
\text { growth }\end{array}$ & $\begin{array}{l}\text { If genomic information is } \\
\text { known, Genome Scale } \\
\text { Metabolic Reconstruction } \\
\text { can be used to construct a } \\
\text { more complete model }\end{array}$ \\
\hline $\begin{array}{l}\text { Constraint-Based Model } \\
\text { with Mixed Integer } \\
\text { Programming introducing } \\
\text { Thermodynamic and } \\
\text { Energetic Constraints }\end{array}$ & $\begin{array}{l}\text { Builds on FBA concepts } \\
\text { and adds thermodynamic } \\
\text { constraints to reduce } \\
\text { solution space }\end{array}$ & $\begin{array}{l}\text { - Similar limitations as } \\
\text { FBA }\end{array}$ \\
\hline Kinetic & $\begin{array}{l}\text { Possible to observe } \\
\text { changes in growth and/or } \\
\text { metabolites over time }\end{array}$ & $\begin{array}{l}\text { - Few kinetic parameters } \\
\text { have been experimentally } \\
\text { determined }\end{array}$ \\
\hline
\end{tabular}


Table 2 Pros and cons of different transformation methods for microalgae

\begin{tabular}{|c|c|c|c|}
\hline & Pros & Cons & Examples \\
\hline Bacterial & $\begin{array}{l}\text { - Requires no special equipment } \\
\text { - Can deliver larger plasmids }\end{array}$ & $\begin{array}{l}\text { - Low transformation } \\
\text { efficiency/limited adaptability } \\
\text { - No genome integration for E. coli } \\
\text { transformation }\end{array}$ & $\begin{array}{l}\text { E. coli delivers plasmids into the } \\
\text { diatoms } P \text {. tricornutum and } T \text {. } \\
\text { pseudonana (Karas et al., 2015) }\end{array}$ \\
\hline Chemical & $\begin{array}{l}\text { - Requires no special equipment } \\
\text { - Inexpensive }\end{array}$ & $\begin{array}{l}\text { - Requires cell wall degradation } \\
\text { - Limited success in a few strains }\end{array}$ & $\begin{array}{l}\text { Polyethylene glycol (PEG) and } \\
\text { DMSO have been used to } \\
\text { transform Chlorella sp (Hawkins } \\
\text { and Nakamura, 1999) }\end{array}$ \\
\hline Electrical & $\begin{array}{l}\text { - Has wide adaptability } \\
\text { - Most protocols do not require } \\
\text { cell wall removal }\end{array}$ & $\begin{array}{l}\text { - Requires electroporator or } \\
\text { programmable power supply } \\
\text { - Only one report of chloroplast } \\
\text { transformation using } \\
\text { electroporation }\end{array}$ & $\begin{array}{l}\text { Nuclear and plastid genome of } P \text {. } \\
\text { tricornutum (Xie et al., 2014) }\end{array}$ \\
\hline Mechanical & $\begin{array}{l}\text { - Can deliver DNA into the } \\
\text { chloroplast } \\
\text { - Requires no cell wall removal } \\
\text { - Glass-bead protocol requires no } \\
\text { special equipment, inexpensive }\end{array}$ & $\begin{array}{l}\text { - Biolistic transformation requires } \\
\text { specialized "gene gun" } \\
\text { - Relatively low transformation } \\
\text { efficiencies }\end{array}$ & $\begin{array}{l}\text { Nuclear and plastid genome of } C \text {. } \\
\text { reinhardtii (Boynton et al., 1988) }\end{array}$ \\
\hline
\end{tabular}


Table 3 Summary of algal genetic engineering work for enhanced TAG production and optimized fatty acid composition

\begin{tabular}{|c|c|c|c|c|}
\hline Protein & $\begin{array}{l}\text { Functional role in FA/TAG } \\
\text { biosynthesis }\end{array}$ & $\begin{array}{l}{ }^{a} \text { Engineered } \\
\text { algae }\end{array}$ & ${ }^{\mathrm{b}}$ Outcome & References \\
\hline \multicolumn{5}{|c|}{ FA synthesis manipulation } \\
\hline ACCase & \multirow[t]{2}{*}{$\begin{array}{l}\text { Generates malonyl-CoA from } \\
\text { acetyl-CoA for FA biosynthesis }\end{array}$} & C. cryptica & $\begin{array}{l}2-3 \text { fold increase in ACC activity, no change in } \\
\text { lipid content }\end{array}$ & $\begin{array}{l}\text { (Dunahay et al., } \\
\text { 1995) }\end{array}$ \\
\hline ACCase & & N. saprophila & $\begin{array}{l}2-3 \text { fold increase in ACC activity, no change in } \\
\text { lipid content }\end{array}$ & $\begin{array}{l}\text { (Dunahay et al., } \\
\text { 1996) }\end{array}$ \\
\hline KAS II & $\begin{array}{l}\text { Thermal regulation of FA } \\
\text { composition of membrane } \\
\text { phospholipids }\end{array}$ & C. reinhardtii & $\begin{array}{l}\text { Increase in C18 fatty acids (lipid quantitation } \\
\text { not known) }\end{array}$ & (Blatti et al., 2013) \\
\hline $\begin{array}{l}\text { Myristyl-ACP } \\
\text { TE }\end{array}$ & \multirow{3}{*}{$\begin{array}{l}\text { Hydrolyze acyl-ACP to release } \\
\text { free FAs; control carbon chain } \\
\text { length and properties of the final } \\
\text { fatty alkane product }\end{array}$} & P. tricornutum & $15 \%$ increase in C $14: 0$ & $\begin{array}{l}\text { (Radakovits et al., } \\
\text { 2011) }\end{array}$ \\
\hline Lauryl-ACP TE & & P. tricornutum & $6.2 \%$ increase in $\mathrm{C} 12: 0$ & $\begin{array}{l}\text { (Radakovits et al., } \\
\text { 2011) }\end{array}$ \\
\hline $\begin{array}{l}\text { Lauryl-ACP TE } \\
\text { and FatB1 TE }\end{array}$ & & C. reinhardtii & No significant change in fatty acid profile & (Blatti et al., 2013) \\
\hline FAB2 & $\begin{array}{l}\text { Catalyzes the conversion of } \\
\text { stearic acid }(18: 0) \text { to oleic acid } \\
(18: 1)\end{array}$ & C. reinhardtii & $\begin{array}{l}28 \% \text { increase in total fatty acid content with a } \\
2.4 \text {-fold increase in oleic acid }(18: 1)\end{array}$ & $\begin{array}{l}\text { (Hwangbo et al., } \\
\text { 2014) }\end{array}$ \\
\hline $\begin{array}{c}\text { NoD12 }(\Delta 12- \\
\text { desaturase })\end{array}$ & $\begin{array}{l}\text { Catalyzes synthesis of linoleic } \\
\text { acid from oleic acid, the } \\
\text { committed step of long chain }\end{array}$ & N. oceanica & $\begin{array}{l}50 \%-75 \% \text { increase in arachidonic acid }(20: 4 \mathrm{n} \\
-6) \text { in TAG }\end{array}$ & (Kaye et al., 2015) \\
\hline
\end{tabular}




\begin{tabular}{|c|c|c|c|c|}
\hline & $\begin{array}{l}\text { polyunsaturated fatty acid } \\
\text { biosynthesis }\end{array}$ & & & \\
\hline \multicolumn{5}{|c|}{ Kennedy pathway enzymes } \\
\hline DGAT2 & \multirow{5}{*}{$\begin{array}{l}\text { Catalyzes addition of an acyl } \\
\text { group to the sn-3 position of } \\
\text { DAG, a rate-limiting step of } \\
\text { TAG synthesis }\end{array}$} & P. tricornutum & $\begin{array}{l}35 \% \text { increase in neutral lipid and } 76.2 \% \\
\text { increase in EPA }\end{array}$ & (Niu et al., 2013) \\
\hline $\begin{array}{l}\text { DGAT2-1; } \\
\text { DGAT2-5 }\end{array}$ & & C. reinhardtii & $\begin{array}{l}20 \% \text { and } 44 \% \text { increase of total lipid content in } \\
\text { the DGAT } 2-1 \text { and DGAT2-5 overexpression } \\
\text { lines, respectively }\end{array}$ & (Deng et al., 2012) \\
\hline DGAT2-a,b,c & & C. reinhardtii & No significant change in fatty acid profiles & $\begin{array}{l}\text { (La Russa et al., } \\
\text { 2012) }\end{array}$ \\
\hline DGAT2 & & T. pseudonana & $\begin{array}{l}1.52 \text { to } 1.95 \text {-fold increase in TAG accumulation } \\
\text { without detriment to growth }\end{array}$ & $\begin{array}{l}\text { (Manandhar- } \\
\text { Shrestha and } \\
\text { Hildebrand, 2015) }\end{array}$ \\
\hline DGAT4 & & C. reinhardtii & About 1.5 to 2.5 -fold increase in TAG content & (Iwai et al., 2014) \\
\hline $\begin{array}{l}\text { G3PDH + GPAT } \\
+ \text { LPAT + PAP + } \\
\quad \text { DGAT }\end{array}$ & $\begin{array}{l}\text { Five-gene cassette encoding } \\
\text { Kennedy pathway enzymes }\end{array}$ & C. minutissima & Two-fold increase in TAG content & (Hsieh et al., 2012) \\
\hline PDAT1 & $\begin{array}{l}\text { Catalyzes conversion of } \\
\text { phospholipids into TAG }\end{array}$ & C. reinhardtii & $\begin{array}{l}25 \% \text { decrease in TAG content in the insertional } \\
\text { mutant (knockout) of PDAT } 1\end{array}$ & (Boyle et al., 2012) \\
\hline GPAT & $\begin{array}{l}\text { Catalyzes the first committed } \\
\text { step of the Kennedy pathway }\end{array}$ & C. reinhardtii & $\begin{array}{l}50 \% \text { increase in the TAG content without } \\
\text { compromising growth }\end{array}$ & $\begin{array}{l}\text { (Iskandarov et al., } \\
\text { 2015) }\end{array}$ \\
\hline
\end{tabular}




\begin{tabular}{|c|c|c|c|c|}
\hline $\begin{array}{l}\text { MLDP (major } \\
\text { lipid droplet } \\
\text { protein) }\end{array}$ & $\begin{array}{l}\text { Implicated in lipid droplet } \\
\text { formation or maintenance }\end{array}$ & C. reinhardtii & $\begin{array}{l}\text { Increased lipid droplet size with no changes in } \\
\text { TAG content in the MLDP knockdown lines }\end{array}$ & $\begin{array}{l}\text { (Moellering and } \\
\text { Benning, 2009) }\end{array}$ \\
\hline PNPLA3 & $\begin{array}{l}\text { Membrane bound protein } \\
\text { associated with lipid droplets }\end{array}$ & P. tricornutum & $70 \%$ increase in neutral lipid content & (Wang et al., 2015) \\
\hline $\begin{array}{l}\text { Nitrogen } \\
\text { regulatory } \\
\text { protein PII } \\
\text { protein }\end{array}$ & $\begin{array}{l}\text { Negatively controls TAG } \\
\text { accumulation in LBs during } \\
\text { acclimation to nitrogen } \\
\text { starvation }\end{array}$ & C. reinhardtii & $\begin{array}{l}\text { Over-accumulation of LBs and a } 1.5 \text {-fold } \\
\text { increase in TAG level in the PII knockdown } \\
\text { lines }\end{array}$ & $\begin{array}{l}\text { (Zalutskaya et al., } \\
\text { 2015) }\end{array}$ \\
\hline \multicolumn{5}{|c|}{ Citric acid cycle enzymes } \\
\hline $\begin{array}{l}\text { Phosphoenolpyru } \\
\text { vate carboxylase } \\
\quad 1, \text { PEPC1 }\end{array}$ & $\begin{array}{l}\text { Channelizes carbon flux coming } \\
\text { from glycolysis towards protein } \\
\text { or FA }\end{array}$ & C. reinhardtii & $\begin{array}{l}20 \% \text { increase in TAG level in the } P E P C 1 \\
\text { knockdown lines }\end{array}$ & (Deng et al., 2014) \\
\hline $\begin{array}{l}\text { Citrate synthase } \\
\text { (CIS) }\end{array}$ & $\begin{array}{l}\text { Regulates carbon flux to FA } \\
\text { synthesis or the TCA cycle }\end{array}$ & C. reinhardtii & $\begin{array}{l}\text { A } 169.5 \% \text { increase in TAG content in the } C I S \\
\text { knockdown lines }\end{array}$ & (Deng et al., 2013) \\
\hline \multicolumn{5}{|c|}{ Kinases and transcription factors } \\
\hline $\mathrm{NAD}(\mathrm{H})$ kinase & Catalyzes synthesis of NADPH & C. pyrenoidosa & $\begin{array}{l}110.4 \% \text { increase in cellular lipid content without } \\
\text { reducing growth rate }\end{array}$ & (Fan et al., 2015) \\
\hline NRR1 & $\begin{array}{l}\text { Associated with transcriptional } \\
\text { regulation of lipid biosynthetic } \\
\text { genes under nitrogen-deprivation } \\
\text { conditions }\end{array}$ & C. reinhardtii & $\begin{array}{l}50 \% \text { decrease in TAG content in the insertional } \\
\text { mutant of nrr1-1 under nitrogen-deprivation }\end{array}$ & (Boyle et al., 2012) \\
\hline $\begin{array}{l}\text { DOF-type } \\
\text { transcription } \\
\text { factor }\end{array}$ & $\begin{array}{l}\text { Transcriptional regulator for } \\
\text { genes involved in FA and } \\
\text { glycerolipid biosynthesis }\end{array}$ & C. reinhardtii & 2-fold increase of total lipids & $\begin{array}{l}\text { (Ibanez-Salazar et } \\
\text { al., 2014) }\end{array}$ \\
\hline
\end{tabular}


${ }^{a}$ species abbreviations: Cyclotella cryptica, Navicula saprophila, Phaeodactylum tricornutum, Thalassiosira pseudonana (diatoms); Chlamydomonas reinhardtii, Chlorella minutissima, Chlorella pyrenoidosa (green algae); Nannochloropsis oceanica

${ }^{b}$ engineering outcome in the respective gene overexpression lines unless otherwise stated. 


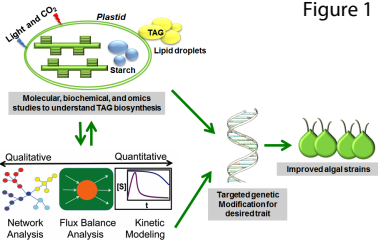

Computational model prediction of ratelimiting steps for TAG production 


\section{Figure 2}

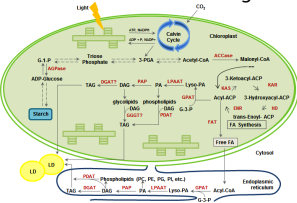

